Aus der Abteilung Allgemeinmedizin

(Prof. Dr. med. M. M. Kochen, MPH, FRCGP)

im Zentrum Innere Medizin

der Medizinischen Fakultät der Universität Göttingen

\title{
Evidenz-basierte Pharmakotherapie der Herzinsuffizienz an der stationär-ambulanten Schnittstelle
}

\author{
INAUGURAL - DISSERTATION \\ zur Erlangung des Doktorgrades \\ der Medizinischen Fakultät \\ der Georg-August-Universität zu Göttingen \\ vorgelegt von \\ Cordula Sobek \\ aus \\ Halle/Saale
}

Göttingen 2007 
De k a n:

I. Berichterstatter:

II. Berichterstatter/in:

III. Berichterstatter/in:
Prof. Dr. med. C. Frömmel

Prof. Dr. med. M. M. Kochen, MPH, FRCGP

Tag der mündlichen Prüfung: 
$\begin{array}{lll}\text { 1. Einleitung } & 6\end{array}$

$\begin{array}{lll}2 & \text { Stand der Forschung } & 8\end{array}$

2.1 Herzinsuffizienz 8

2.1.1 Definition und Pathogenese 8

2.1.2 Pathophysiologie 8

2.1.3 Epidemiologie 9

2.1.4 Klinik und Einteilung 9

$\begin{array}{lll}2.1 .5 & \text { Diagnostik } & 10\end{array}$

2.1.6 Therapie 11

2.2 Probleme der Versorgung herzinsuffizienter Patienten 14

2.2.1 Hausarzt 14

2.2.2 Klinik 17

$\begin{array}{lll}2.2 .3 & \text { Schnittstellenproblematik } & 18\end{array}$

3. Fragestellung und Ziel $\quad 21$

4. Material und Methoden 22

4.1 Patienten 22

4.2 Datenerhebung 23

$4.3 \quad$ Dateneingabe 25

4.4 Datenschutz und Genehmigung durch die Ethik-Kommission 25

$\begin{array}{ll}\text { 5. Ergebnisse } & 26\end{array}$

5.1. Patientencharakteristika 26

5.1.1 Geschlechterverteilung, Alter, Aufenthaltsdauer 26

5.1.2 NYHA - Stadien 26

$\begin{array}{lll}5.1 .3 & \text { Ejektionsfraktion } & 27\end{array}$

5.1.4 Grunderkrankungen und diagnostisch-therapeutische Maßnahmen 28

5.2 Pharmakotherapie der Herzinsuffizienz 29

5.2.1 Verordnung von ACE-Hemmern 31

5.2.2 Verordnung von AT-II-Antagonisten 31

5.2.3 Verordnung von evidenzbasierten Betablockern 32

5.2.4 Verordnung von Aldosteronantagonisten 32 
5.2.5 Medikamentenwechsel an der ambulant-stationären Schnittstelle

5.2.6 Medikamentenwechsel an der stationär-ambulanten Schnittstelle

5.3 Nichtmedikamentöse Therapie der Herzinsuffizienz 35

$\begin{array}{lll}6 . & \text { Diskussion } & 38\end{array}$

$\begin{array}{lll}6.1 & \text { Limitationen } & 38\end{array}$

$\begin{array}{lll}6.2 & \text { Kontext } & 39\end{array}$

6.3 Bedeutung für die Praxis 44

6.4 Bedeutung für die weitere Forschung 45

$\begin{array}{lll}6.5 & \text { Ausblick } & 46\end{array}$

$\begin{array}{lll}\text { 7. Zusammenfassung } & 47\end{array}$

$\begin{array}{lll}\text { 8. Anhang } & 49\end{array}$

8.1 Dokumentationsbogen 49

8.2 Fragebogen Telefoninterview 52

8.3 Fax an Hausärzte $\quad 54$

$\begin{array}{ll}\text { 9. Literaturverzeichnis } & 55\end{array}$ 


\section{Verzeichnis der Abbildungen und Tabellen}

Seite

$\begin{array}{llr}\text { Abbildung 1: } & \text { Ablaufdiagramm zur Studienteilnahme } & 24 \\ \text { Diagramm 1a: } & \text { NYHA-Stadien bei Rekrutierung } & 27 \\ \text { Diagramm 1b: } & \text { NYHA-Stadien 14 Tage nach Entlassung } & 27 \\ \text { Diagramm 2: } & \text { Ejektionsfraktion - NYHA-Stadium } & 28 \\ \text { Diagramm 3: } & \begin{array}{l}\text { Verordnung von ACE-Hemmern in Abhängigkeit vom } \\ \text { Schweregrad nach NYHA }\end{array} & 31\end{array}$

Diagramm 4: Verordnung von Aldosteronantagonisten in Abhängigkeit 33 vom Schweregrad nach NYHA

$\begin{array}{lll}\text { Diagramm 5: } & \text { Grippeimpfung } & 36\end{array}$

$\begin{array}{lll}\text { Diagramm 6: } & \text { Pneumokokkenimpfung } & 37\end{array}$

$\begin{array}{lll}\text { Tabelle 1: } & 10\end{array}$

Tabelle 2: $\quad$ Prästationäre, stationäre und poststationäre Pharma- $\quad 30$ kotherapie der Herzinsuffizienz

Tabelle 3: $\quad$ Medikamentenwechsel im Krankenhaus 34

Tabelle 4: $\quad$ Medikamentenwechsel 14 Tage poststationär 35 


\section{Einleitung}

Die Herzinsuffizienz stellt bezüglich der Diagnostik- und Therapieentscheidungen hohe Anforderungen an Hausärzte und Kliniker. Sie erlaubt keinesfalls ein einfaches Routinevorgehen. Deshalb sind in interdisziplinärer Zusammenarbeit auf internationalem und nationalem Gebiet von mehreren Fachgesellschaften Therapieempfehlungen zur Behandlung der Herzinsuffizienz erarbeitet worden (ACC/AHA Task Force on Practice Guidelines 2001, Hoppe und Erdmann 2001, www.evidence.de 2005, www.uniduesseldorf.de/WWW/AWMF 2005, www.pmvforschungsgruppe.de 2006).

Scheinbar nicht einfach ist deren erfolgreiche Implementation in der täglichen Praxis. Noch haben sie nicht den Stellenwert erreicht, der für eine optimale Betreuung notwendig wäre. Ursachen für eine mangelnde Leitlinientreue in der Praxis (hausärztliche Versorgung) sind die Anwendung traditioneller Therapiekonzepte, Unsicherheiten in der Diagnostik und Kommunikationsbarrieren zwischen Hausärzten und Kliniken (Böger 2002, Phillips et al. 2004, Greer 1988, Kanouse und Jacoby 1988). In bestimmten Fällen entscheiden sich Hausärzte allerdings bewusst gegen die Einhaltung von Leitlinien („,best practices"). Faktoren dafür sind die individuelle Non-Compliance der Patienten, Patientenalter und -geschlecht, Ausmaß der Komorbidität sowie die Behandlungspräferenz der Ärzte (Mottur-Pilson et al. 2001).

ACE-Hemmer und ersatzweise AT-II-Antagonisten gehören zum Therapiestandard in allen NYHA-Gruppen, werden aber von Hausärzten seltener verordnet als von Kardiologen und teilweise nicht ausreichend dosiert (www.pmvforschungsgruppe.de 2006, Bungard et al. 2001, Baker et al. 1999, Cline et al. 2002, McMullan und Silke 2001). Die Verschreibungszahlen für Betablocker mit nachgewiesener Prognoseverbesserung erreichen bei Hausärzten in der Behandlung der Herzinsuffizienz noch nicht die erwünschten Häufigkeiten (Phillips et al. 2004). Befürchtungen bezüglich Nebenwirkungen und Kontraindikationen spielen hierbei eine wesentliche Rolle (Hickling et al. 2001). Während für alle ACE-Hemmer (Hall et al. 1997, Hood et al. 1991) und AT-IIAntagonisten (McKelvie et al. 1999, Pitt et al. 2000) ein prognoseverbessernder Effekt als gesichert gilt, trifft dies nachweislich nur auf drei der gängigen Betablocker zu: Metoprolol (MERIT-HF Study Group 1999), Bisoprolol (CIBIS-II-Research-Group 1999) und Carvedilol (Lechat et al. 1998). Neben den ACE-Hemmern, Aldosteronantagonisten und 
Beta-Blockern werden in der vorliegenden Arbeit auch die in ihrem Einsatz noch unterrepräsentierten Aldosteronantagonisten und Probleme der nichtmedikamentösen Therapie betrachtet (McMurray et al. 2001). 


\section{Stand der Forschung}

\subsection{Herzinsuffizienz}

\subsubsection{Definition und Pathogenese}

Die Herzinsuffizienz bezeichnet einen pathophysiologischen Zustand, der aus einer beeinträchtigten Funktion des Herzens resultiert, das nicht mehr in der Lage ist, den metabolischen Erfordernissen der Gewebe angepasste Blutmengen auszuwerfen (Task Force for the Diagnosis and Treatment of Chronic Heart Failure, European Society of Cardiology 2001). Man unterscheidet die (1) akute und (2) chronische Form der Herzinsuffizienz.

1) Die akute Herzinsuffizienz kann sich aus einer dekompensierten chronischen Herzinsuffizienz heraus entwickeln oder entsteht in Folge eines Lungenödems, eines Herzinfarkts oder einer hypertensiven Krise (McMurray und Stewart 2000).

2) Die häufigste Ursache der chronischen Herzinsuffizienz ist die koronare Herzkrankheit, allein oder in Kombination mit einer Hypertonie. Seltenere Ursachen sind Diabetes, Herzvitien und Kardiomyopathien.

\subsubsection{Pathophysiologie}

Nach einer myokardialen Schädigung (Druck- / Volumenbelastung, Infarkt) kommt es zu einer Myozytenhypertrophie, zum remodeling (myokardialer Umbau) und zur Ventrikeldilatation mit dem Ziel, ein genügendes Schlagvolumen aufrechtzuerhalten. Apoptosevorgänge am dilatierten Herzen bedingen einen weiteren Abfall der Pumpfunktion und führen zur Aktivierung neuroendokriner Systeme. Es erfolgt eine Stimulation des Sympathikus, des Renin-Angiotensin-Aldosteron-Systems und eine vermehrte Ausschüttung von Zytokinen. Daraus resultieren eine periphere Vasokonstriktion, eine Flüssigkeitsretention sowie eine erhöhte Arrhythmieneigung. Diese Kompensationsmechanismen bilden einen Circulus vitiosus, der (wenn er nicht durchbrochen werden kann) ein Fortschreiten der Erkrankung begünstigt (Arzneimittelkommission der deutschen Ärzteschaft 2001, Task force for the Diagnosis and Treatment of Chronic Heart Failure, European Society of Cardiology 2001). 


\subsubsection{Epidemiologie}

Die Prävalenz der Herzinsuffizienz nimmt mit steigendem Alter zu. Die Prävalenz in der Patientengruppe $\leq 65$ Jahre liegt im Bereich zwischen $0,4 \%$ bis $2 \%$. Bei der Patientengruppe $\geq 65$ Jahre steigt die Prävalenz bis auf $8 \%$ an. Zusätzlich konnte eine Zunahme der Prävalenz in den letzten Jahrzehnten verzeichnet werden, was auf das sich erhöhende Durchschnittsalter der Gesellschaft zurückzuführen ist. Die Langzeitprognose der Herzinsuffizienz ist ungünstig und mit der von Malignomen vergleichbar (McMurray und Stewart 2000). Prädiktoren, die die Mortalität der Herzinsuffizienz beeinflussen, sind (Lee et al. 2003):

- Alter

- Blutdruck

- Hyponatriämie

- eingeschränkte Nierenfunktion

- Demenz

- Leberzirrhose

- Tumorerkrankungen.

\subsubsection{Klinik und Einteilung}

Klinische Symptome der Herzinsuffizienz sind: Dyspnoe, Nykturie, Müdigkeit und Leistungsminderung. Anhand des Leitsymptoms Dyspnoe wird der Schweregrad der Herzinsuffizienz international entsprechend der Klassifikation der New York Heart Association (NYHA) abgeschätzt. Die Einteilung erfolgt in die Stadien I bis IV.

In der klinischen Untersuchung können sich Ödeme abhängiger Körperpartien, Tachykardie, Hepatomegalie, hepatojugulärer Reflux, Aszites, Jugularvenenstauung, pulmonale Rasselgeräusche, Kardiomegalie und ein 3. Herzton finden (www.dgk.org 2005). 
Tabelle 1: NYHA-Stadien (www.dgk.org 2005)

\begin{tabular}{|l|l|}
\hline $\begin{array}{l}\text { NYHA- } \\
\text { Stadium }\end{array}$ & Beschwerden \\
\hline I & $\begin{array}{l}\text { Herzerkrankung ohne körperliche Limitationen. Allägliche körperliche } \\
\text { Belastung verursacht keine inadäquate Erschöpfung, Rhythmusstö- } \\
\text { rungen, Luftnot oder Angina pectoris. }\end{array}$ \\
\hline II & $\begin{array}{l}\text { Herzerkrankung mit leichter Einschränkung der körperlichen Leis- } \\
\text { tungsfähigkeit. Keine Beschwerden in Ruhe. Alltägliche körperliche } \\
\text { Belastung verursacht Erschöpfung, Rhythmusstörungen, Luftnot oder } \\
\text { Angina pectoris. }\end{array}$ \\
\hline III & $\begin{array}{l}\text { Herzerkrankung mit höhergradiger Einschränkung der körperlichen } \\
\text { Leistungsfähigkeit bei gewohnter Tätigkeit. Keine Beschwerden in } \\
\text { Ruhe. Geringe körperliche Belastung verursacht Erschöpfung, } \\
\text { Rhythmusstörungen, Luftnot oder Angina pectoris. }\end{array}$ \\
\hline IV & $\begin{array}{l}\text { Herzerkrankung mit Beschwerden bei allen körperlichen Aktivitäten } \\
\text { und in Ruhe. Bettlägerigkeit. }\end{array}$ \\
\hline
\end{tabular}

\subsubsection{Diagnostik}

Anamnese und klinische Untersuchung (s. o.) sind wegweisend. Bei entsprechendem Verdacht sollten sich technische Untersuchungen anschließen, um eine adäquate und gegebenenfalls kausale Therapie der Herzinsuffizienz durchführen zu können. Es können eine Standard-Laboruntersuchung (Blutbild, Serum-Elektrolyte, Serum-Kreatinin, Serumlipide, Nüchtern-Blutzucker, Leberenzyme, Urinstatus, CRP) sowie ein EKG und ein Röntgen-Thorax durchgeführt werden. Ergänzende Laborparameter bei spezieller Indikation können Troponin T/CK/CK-MB, BNP, Schilddrüsenhormone, Harnstoff, Harnsäure und Gerinnungsparameter sein (Hoppe 2005).

Einen besonderen Stellenwert besitzt die Echokardiografie. Sie erlaubt die Beurteilung der kardialen Dysfunktion, bei gleichzeitiger geringster Belastung für den Patienten, nichtinvasiver Durchführbarkeit und guter Verfügbarkeit. Die Echokardiografie wird zur Diagnosestellung, Schweregradbeurteilung und Verlaufsbeobachtung der Herzinsuffi- 
zienz eingesetzt. Es können Kontraktionsstörungen, Dilatationen, systolische oder diastolische Funktionsstörungen sowie kardiale Vitien dargestellt und quantifiziert werden. Von besonderer Bedeutung ist die Möglichkeit, die Ejektionsfraktion des linken Ventrikels bei Patienten mit systolischer Herzinsuffizienz zu bestimmen. Eine weiterreichende Diagnostik erfolgt zum Beispiel bei Verdacht auf eine koronare Herzkrankheit mit Hilfe einer Stressechokardiografie und der Durchführung einer Koronarangiografie (Task force for the Diagnosis and Treatment of Chronic Heart Failure, European Society of Cardiology 2001; www.dgk.org 2005).

\subsubsection{Therapie}

Die Therapie der chronischen Herzinsuffizienz verfolgt mehrere Ziele gleichzeitig. Dazu gehören:

- Senkung der Letalität

- Hemmung der Krankheitsprogression

- Besserung der Beschwerdesymptomatik

- Senkung der Hospitalisationsrate

- Verbesserung hämodynamischer Parameter.

Um diese Ziele umzusetzen, stehen sowohl (1) medikamentöse als auch (2) nichtmedikamentöse Therapieoptionen zur Verfügung. Beide soltten in einem modernen Therapieschema Anwendung finden, um ergänzende und synergistische Effekte beider Therapiearme effektiv zu nutzen. Diese Therapien werden von zahlreichen Leitlinien empfohlen, wie zum Beispiel in der Leitlinie zur Therapie der Herzinsuffizienz der European Society of Cardiology (Task force for the Diagnosis and Treatment of Chronic Heart Failure, European Society of Cardiology 2001). Aktuell konnte durch Komajda et al. (2005) gezeigt werden, dass die Durchführung einer leitlinienadhärenten Therapie mit reduzierten Hospitalisierungsraten und einem verlängerten Zeitintervall bis zum nächsten Krankenhausaufenthalt einhergeht.

1) Bei der medikamentösen Therapie stehen a) ACE-Hemmer bzw. Angiotensin-IIAntagonisten, b) Beta-Blocker und c) Aldosteronantagonisten im Zentrum der Betrachtung. 
a) Jeder Patient mit einer systolischen Herzinsuffizienz (NYHA I - IV) sollte einen ACE-Hemmer erhalten. In kontrollierten Studien konnte für diese Medikamentengruppe gezeigt werden, dass sie die Symptomatik und Belastungstoleranz der Patienten verbessert, die Hospitalisationsrate aufgrund der Herzinsuffizienz vermindert und die Letalität signifikant senkt (Cohn et al. 1991; The SOLVD investigators 1991; The CONSENSUS Trial Study Group 1987). Auch für Patienten, die infolge eines Herzinfarktes herzinsuffizient werden, konnte eine Verbesserung der Überlebensrate sowie der Reinfarktrate und der Häufigkeit der Krankenhauseinweisung gezeigt werden (Flather et al. 2000; Hall et al. 1997; ISIS-4 Collaborative Group 1995; Kober et al. 1995; Pfeffer et al. 1992). Bei der Dosierung des ACE-Hemmers sollte neben der Verbesserung der Symptomatik das Erreichen der Zieldosis angestrebt werden. Hierfür konnte in der ATLAS-Studie belegt werden, dass bei gleicher Nebenwirkungshäufigkeit die Gesamtletalität tendenziell und die kardiovaskuläre Letalität sowie die Hospitalisationsrate signifikant geringer ausfällt (Packer et al. 1999). Als Nebenwirkungen können Husten oder ein Angioödem auftreten. Absolute Kontraindikationen sind beidseitige Nierenarterienstenosen, Serum-Kalium $>5,5 \mathrm{mmol} / \mathrm{l}$, chronische Niereninsuffizienz mit GFR $<30 \mathrm{ml} / \mathrm{min}$, eine hypertrophe Obstruktion des linksventrikulären Ausflusstraktes, Herzklappenstenosen, ein Angioödem im Rahmen früherer Therapien mit ACE-Hemmern, Schwangerschaft und symptomatische Hypotension (Arzneimittelkommission der deutschen Ärzteschaft 2001, www.evidence.de 2005).

Die Angiotensin-II-Rezeptorantagonisten finden heute vorwiegend Einsatz bei Patienten mit einer ACE-Hemmer-Unverträglichkeit im NYHA-Stadium II - IV, da bei innen Nebenwirkungen deutlich seltener auftreten (Burnier und Brunner 2000). Im Vergleich mit Placebo führte Candesartan zu einer deutlicheren Verbesserung des NYHA-Stadiums (32,5\% versus 35,4\%) (O'Meara et al. 2004). In der VAL-HeFT-Studie (Valsartan Heart Failure Trial) konnte gezeigt werden, dass Valsartan, zusätzlich zu einer Standardtherapie gegeben (die in fast allen Fällen einen ACE-Hemmer einschloss), die Hospitalisationsrate senkte, nicht jedoch die Gesamtmortalität (Cohn et al. 1999). 
b) Ergänzend zur ACE-Hemmer-Basismedikation wird heute die Therapie mit BetaBlockern für Patienten im NYHA-Stadium II - IV empfohlen. Für Metoprolol, Bisoprolol und Carvedilol konnte in verschiedenen großen, randomisierten, Plazebo-kontrolllierten Endpunkt-Studien gezeigt werden, dass sie die kardiovaskuläre Mortalität, wie auch die Gesamtmortalität und die Rate des plötzlichen Herztods reduzieren (MERIT-HF Study Group 1999, CIBIS-II-Research-Group 1999, Lechat et al. 1998). Die Therapie sollte einschleichend mit einem der oben genannten Beta-Blocker (ohne intrinsische Aktivität) beginnen und nur bei Patienten mit einer stabilen Herzinsuffizienz erfolgen, da es initial zur Abnahme der Auswurffraktion des Herzens und zum Blutdruckabfall kommt. Auch die Beta-Blocker sollten auf die in den Studien genannten Zieldosen auftitriert werden.

c) Der Einsatz von Aldosteronantagonisten wird heute begleitend zu einer ACEHemmer- und Diuretikatherapie für die NYHA-Stadien III - IV empfohlen. Es konnten hierfür eine Verbesserung der Symptomatik, eine Reduktion der Hospitalisationshäufigkeit, eine Senkung der Herztodesrate und der Letalität infolge Pumpversagens gezeigt werden (Pitt et al. 1999, Ramires et al. 2000). Aldosteronantagonisten können zur Therapie einer persistenten Hypokaliämie eingesetzt werden. Als unerwünschte Nebenwirkung kann in 10\% der Fälle eine Gynäkomastie auftreten, die das Absetzen des Medikamentes erzwingt.

2) Zentrale Punkte der nichtmedikamentösen Therapie sind (Arzneimittelkommission der deutschen Ärzteschaft 2001):

- Reduktion kardiovaskulärer Risikofaktoren (z. B. Cholesterin, Rauchen, Alkohol)

- Normalisierung des Gewichts

- Beschränkung der Natriumzufuhr auf unter $3 \mathrm{~g} / \mathrm{Tag}$

- Beschränkung der Flüssigkeitszufuhr auf $\leq 2 \mathrm{l} / \mathrm{Tag}$

- tägliche Gewichtskontrolle durch den Patienten und Aufklärung zum weiteren Verhalten (z. B. Konsultieren eines Arztes)

- körperliches Training bei kompensierter Herzinsuffizienz, Schonung/Bettruhe in Phasen der Dekompensation. 
Die Umsetzung dieser Punkte kann helfen, einer Dekompensation der chronischen Herzinsuffizienz vorzubeugen. Es konnte gezeigt werden, dass mehr als zwei Drittel der herzinsuffizienten Patienten über nur ungenügendes Basiswissen (regelmäßige Gewichtskontrolle, Diät, Verhalten bei Symptomzunahme) bezüglich ihrer Erkrankung verfügen (Böger 2002, Wagdi et al. 1993). Knapp die Hälfte der Patienten war sich nicht der Bedeutung einer täglichen Gewichtskontrolle bewusst. Immerhin 80\% der Patienten wussten, dass sie ihre Salzzufuhr limitieren sollten, aber nur ein Drittel vermied salziges Essen. Weiterhin glaubte ein Drittel der Patienten, sie sollten viel trinken (Ni et al. 1999).

Die unzureichende Patienteninformation bedingt somit eine ebenfalls ungenügende Patientencompliance und begünstigt das Auftreten einer Dekompensation. Ein höherer Wissensstand war assoziiert mit zurückliegenden Krankenhausaufenthalten und dem Erhalt von Informationen zum Selbstmanagement durch Ärzte und/oder Arzthelferinnen. Verheiratete Patienten waren im Schnitt ebenfalls besser informiert. Im Bereich der nichtmedikamentösen Therapie liegt somit ein wichtiges Potenzial zur Senkung der Hospitalisationsfrequenz (Ni et al. 1999, Wagdi et al. 1993).

\subsection{Probleme der Versorgung herzinsuffizienter Patienten}

\subsubsection{Hausarzt}

In der Langzeitbetreuung sind Hausärzte vorwiegend mit älteren herzinsuffizienten Patienten konfrontiert (Durchschnittsalter MERIT-HF Studie: 64 Jahre [Wikstrand et al. 2003]), die durch eine eingeschränkte Mobilität auch weniger diagnostische Maßnahmen erhalten können (Rutten et al. 2003). Der frühe Einsatz von ACE-Hemmern im hausärztlichen Setting ist bei asymptomatischen Patienten schwer realisierbar (Chin et al. 1997). Bei symptomatischen Patienten werden sie häufiger als bei asymptomatischen Patienten verordnet. Zieldosen, wie zum Beispiel in den Guidelines der European Society of Cardiology (Task Force for the Diagnosis and Treatment of Chronic Heart Failure, European Society of Cardiology 2001) empfohlen, werden nur in 50\% erreicht (Cleland et al. 2002). Autoren zweier britischer Arbeiten (Horne et al. 1999, McColl et al. 1998) konnten in strukturierten Interviews folgende Barrieren einer optimalen Versorgung von Herzinsuffizienzpatienten in Hausarztpraxen identifizieren: 
- Diuretika werden als besonders nützlich angesehen und deshalb bevorzugt eingesetzt.

- Das Nebenwirkungspotenzial von ACE-Hemmern wird überschätzt.

- Leitlinien orientieren sich häufig nicht an den Erfordernissen der hausärztlichen Praxis.

Die Langzeitbetreuung chronisch herzinsuffizienter Patienten wird in erster Linie durch den Hausarzt geleistet. Die regelmäßigen Kontakte zwischen Hausarzt und Patient gewährleisten eine kontinuierliche Erhebung des aktuellen Status und einer eventuell daraus resultierenden Anpassung der Therapie. So konnten Autoren einer schwedischen Studie zeigen, dass die Patienten im Durchschnitt sieben Mal im Jahr mit ihrem Hausarzt in Kontakt stehen (Nilsson und Strender 2002).

Schwierigkeiten in der Umsetzung geplanter Therapien ergeben sich im Rahmen der Grunderkrankungen und durch das Vorliegen zusätzlicher Begleiterkrankungen (Multimorbidität). Die betroffenen Patienten nehmen oft schon zahlreiche Medikamente ein. Somit ist die Bereitschaft zu weiteren Medikamenteneinnahmen reduziert. Gleichzeitig ist die Angst vor Medikamenteninteraktionen erhöht. So zeigte eine amerikanische Studie einen Zusammenhang zwischen Lebensalter und der Prävalenz des Auftretens von Diabetes mellitus (38\%), chronischen Lungenerkrankungen (33\%) und Vorhofflimmern (30\%) (Masoudi und Krumholz 2003). In einer schwedischen Studie lagen in 37,2\% der Fälle eine ischämische Herzerkrankung und in $27,3 \%$ der Fälle ein Hypertonus vor (Nilsson und Strender 2002).

Erschwerend auf die Versorgung herzinsuffizienter Patienten wirken sich mitunter psychische Kosymptome aus, die eine Anpassung der medikamentösen und nichtmedikamentösen (Wiegen, salzarme Ernährung) Therapie erfordern und zu Complianceproblemen führen können. Die Modifikation des Lebensstils sowie die Schaffung eines gesundheitsbezogenen Problembewusstseins (Nutzung von Influenza- und Pneumokokkenimpfung - www.evidence.de 2005, www.nice.org.uk 2005) sind häufig schwierig und zeitaufwendig. Eine amerikanische Studie beschreibt drei Hauptgründe, die Patienten für Schwierigkeiten in der Umsetzung der kochsalzarmen Ernährung angeben (Bentley et al. 2005): 
- mangelndes Wissen

- Konflikte bei salzreicher Ernährungsweise der Familienmitglieder

- begrenzte Speisenauswahl.

Selbst das Mitwirken geschulter Arzthelferinnen mit dem Ziel, das Selbstmanagement herzinsuffizienter Patienten zu verbessern, zeigte keinen nennenswerten Effekt (Martensson et al. 2005).

Alter und Komorbidität der Patienten stellen aber nicht nur in der Therapieplanung, sondern auch bei der Diagnosestellung eine Herausforderung dar. Hier gilt es zunächst ohne invasive Untersuchungen die Diagnose einzukreisen, nach alternativen Erkrankungen zu suchen und das individuelle Risiko des Patienten einzuschätzen. Dabei wird besonders der Hausarzt mit frühen Krankheitsstadien (NYHA I) und uncharakteristischen Beschwerdebildern konfrontiert, die zusätzlich durch Begleiterkrankungen überlagert sein können (McColl et al. 1998, Horne et al. 1999). Die Diagnosestellung wird dadurch erschwert und eine leitliniengerechte Therapie verzögert. Häufige Anhaltspunkte für die hausärztliche Diagnose einer Herzinsuffizienz sind:

- bestehende Atemnot (92,5\%)

- Rasselgeräusche der Lunge $(52,5 \%)$

- periphere Ödeme $(55,0 \%)$.

Die Echokardiografie wird im hausärztlichen Bereich zurückhaltend eingesetzt. Als Gründe hierfür werden von Hausärzten in Großbritannien angegeben (Fuat et al. 2003, Hickling et al. 2001):

- begrenzte Verfügbarkeit

- lange Wartezeiten

- mangelnde Zusammenarbeit mit Kardiologen

- Unsicherheiten bei der Interpretation der Ergebnisse

- mangelnde Überzeugung von der Echokardiografie als Goldstandard

- Patientenfaktoren (Logistik, Stresssituation für ältere Patienten)

- abrechnungsbezogene Reglementierungen. 
Der Einsatz serologischer Marker, wie zum Beispiel BNP (B-type natriuretic peptide), ist noch nicht flächendeckend nutzbar, da genaue Grenzwerte und einfach zu verwendende Bedside-Tests fehlen (Adlam et al. 2005).

Bei der Ausrichtung der Therapie liegt der Fokus bei Hausärzten und Kardiologen auf verschiedenen Aspekten, die im Folgenden genannt sind:

- Hausarzt $\rightarrow$ Fehlen von Symptomen

- Kardiologe $\rightarrow$ Erreichen der Zieldosen oder des angestrebten Blutdrucks (Chin et al. 1997).

Im Umgang mit ACE-Hemmern steht bei Hausärzten die Angst vor Nebenwirkungen im Vordergrund. Als weitere Günde für einen zurückhaltenderen Einsatz von ACEHemmern gaben die Hausärzte Diagnoseunsicherheit, mangelnde Sicherheit im Management der Erkrankung, Notwendigkeit des renalen Kontrollmonitorings, finanzielle Einschränkungen und patientenabhängige Faktoren an. Die Angst vor Nebenwirkungen ist auch einer der Gründe für eine zurückhaltendere Dosissteigerung (Hickling et al. 2001). Auch die Vermittlung der Dosissteigerung gegenüber dem Patienten, soweit dieser unter einer niedrigeren Dosierung beschwerdefrei ist, stellt ein Problem dar (Fuat et al. 2003).

\subsubsection{Klinik}

Die Krankenhäuser nehmen eine zentrale Rolle in der Akutversorgung herzinsuffizienter Patienten ein. Ihre Stärke liegt in der Möglichkeit einer raschen und vernetzten Diagnostik. Für die Versorgung chronisch herzinsuffizienter Patienten, bei denen die medikamentöse Therapie und die Patienteninformation im Vordergrund stehen (weniger die schnell verfügbaren Nothilfeketten), konnten keine kliniksabhängigen (Universitätsklinik, Lehr- / Kreiskrankenhaus) signifikanten Versorgungsunterschiede nachgewiesen werden (Butler et al. 2003). 
Nach vorliegenden Studien erhielten $70-87 \%$ der Patienten mit der Entlassungsdiagnose Herzinsuffizienz eine Bestimmung der linksventrikulären Ejektionsfraktion (Nohria et al. 1999). Die Angaben zur Verordnung von ACE-Hemmern reichen von $72 \%$ (Nohria et al. 1999) bis zu 89\% (Weil und Tu 2001). Die in klinischen Studien empfohlenen Zieldosen werden nur in 23\% (Weil und Tu 2001) bzw. 45\% (Nohria et al. 1999) erreicht.

Ein Prädiktor für den Einsatz bestimmter Medikamentengruppen ist das Alter (wie in einer französischen Studie gezeigt werden konnte). In dieser Arbeit wurde die Herzinsuffizienzmedikation im Krankenhaus bei Patienten $\leq 75$ Jahren und $\geq 75$ Jahren verglichen. Hierfür zeigte sich ein signifikant geringerer Einsatz von ACE-Hemmern (48\% versus 63\%) und Beta-Blockern (19\% versus 37\%) in der Gruppe der über 75jährigen. Für diese Patientengruppe konnten längere Aufenthaltsdauern im Krankenhaus nachgewiesen werden (Maison et al. 2005). Diese Daten unterstreichen die Notwendigkeit einer häufigeren Gabe von ACE-Hemmern auch im höheren Alter.

Eine amerikanische Studie zeigte, dass die Qualität der Versorgung im Krankenhaus sich in den Wiederaufnahmeraten innerhalb drei Monaten nach Entlassung widerspiegelt und maßgeblich positiv durch das Einbeziehen von Kardiologen beeinflusst wird (Polanczyk et al. 2001).

\subsubsection{Schnittstellenproblematik}

Der Übergang zwischen hausärztlicher und stationärer Versorgung ist für die Kontinuität einer leitliniengerechten Therapie von besonderer Bedeutung. Änderungen der Medikation treten in beiden Richtungen auf (Hausarzt/Krankenhaus, Krankenhaus/Hausarzt) und werden maßgeblich durch die Vielzahl vergleichbarer Medikamente, die Vermarktungsstrategien der Hersteller, die Kommunikation zwischen Klinik und ambulant tätigen Ärzten (Harder et al. 2005) und die Patientencharakteristika beeinflusst. Für Letztere konnten folgende Unterschiede beschrieben werden:

- allgemeinärztlich behandelte Patienten sind älter,

- allgemeinärztlich behandelte Patienten haben mehr Begleiterkrankungen,

- allgemeinärztlich behandelte Patienten haben weniger schwere kardiale Erkrankungen als die von den Kardiologen therapierten Patienten, 
- kardiologische Patienten weisen eine höhere Mortalität in der frühen Nachbeobachtungsperiode auf,

- die Hospitalisierungsdauer liegt bei den allgemeinmedizinischen Patienten höher.

In Bezug auf Lebensqualität und Therapiezufriedenheit gibt es zwischen der hausärztlichen und der kardiologisch betreuten Gruppe keine Unterschiede (Lowe et al. 2000).

Stationäre Spezialisten haben einen wichtigen Einfluss auf den Einsatz neuer Medikamente in der Primärversorgung (Bijl et al. 1998). So wurden zum Beispiel im Nordosten der Niederlande zwei Drittel der kardiovaskulären Medikamente, die durch den Hausarzt verordnet wurden, durch einen Kardiologen/Internisten initiiert (de Vries et al. 1996). Bei Aufnahme des Patienten in die Klinik werden zahlreiche Medikamente sowohl abgesetzt als auch neu angesetzt (Beers et al. 1989, Omori et al. 1991, Cochrane et al. 1992). Diese Variation in der Anzahl und Auswahl der Präparate kann zu Complianceproblemen und zur Beeinträchtigung des Vertrauensverhältnisses zwischen Patient und Hausarzt führen. Insbesondere eine Reduktion der Krankenhausmedikation durch den Hausarzt kann von Patienten als eine Einschränkung der Behandlungsqualität empfunden werden (Himmel et al. 1996).

Himmel et al. beschrieben 1996 die Veränderungen der Langzeitmedikation bei Einweisung ins Krankenhaus. Hier wurde die Änderung der Langzeitmedikation chronisch kranker, allgemeinärztlicher Patienten bei Einweisung ins Krankenhaus analysiert. So wurden $47 \%$ der 420 hausärztlich verordneten Medikamente beibehalten, 28\% abgesetzt und 25\% der Medikamente ersetzt. 192 Medikamente wurden durch die Klinikärzte neu angesetzt.

Im Rahmen einer weiteren Studie (Himmel et al. 2004) wurden Medikamentenwechsel bei 300 Patienten, die in drei verschiedene Kreiskrankenhäuser in Deutschland (Thüringen) eingewiesen wurden, untersucht. Während des Klinikaufenthalts wurden 36\% der hausärztlichen Medikamente abgesetzt. Nur bei 13 Patienten wurden keine Veränderungen an der Medikation vorgenommen. Für die stationär-ambulante Schnittstelle wurde gezeigt, dass nach Entlassung aus der Klinik zwei Drittel der Entlassungsmedikation ohne Änderung übernommen wurde (Himmel et al. 1996). 
Ein Drittel der Verordnungen wurden durch vergleichbare Substanzen oder Generika ersetzt. 12,5\% der Medikamente wurden abgesetzt. Verglichen mit der Medikamenteneinnahme vor dem Klinikaufenthalt kam es zu einem Zuwachs an verordneten Medikamenten um 18,1\%. Tendenziell werden deutlich mehr Medikamentenverordnungen als an der ambulant-stationären Schnittstelle übernommen, an der nahezu die Hälfte aller Medikamente geändert werden.

Neben dem therapeutischen Aspekt wird in einigen Studien auch der ökonomische Stellenwert von Medikamentenwechseln diskutiert. So konnte in einer niederländischen Studie gezeigt werden, dass Spezialisten teurere Medikamente verordnen und nach Entlassung die Medikamentenkosten um 23\% ansteigen (Bijl et al. 1998). Eine weitere Studie verdeutlicht, dass es nur in wenigen Fällen (10\%) möglich war, nach Entlassung eine qualitativ hochwertige medikamentöse Therapie und eine Kosteneinsparung zu erreichen. Eher kam es zum Auftreten von Versorgungsdefiziten bei ausbleibender Kosteneinsparung. Für die Schnittstellen kann somit kein konsistenter Effekt zwischen Medikationsänderung und Kosteneffektivität beobachtet werden (Weltermann et al. 1997).

Hausärzte beklagen den oft langsamen Informationsfluss und fehlende Erklärungen/Begründungen der in der Klinik geänderten Medikation (aus denen man bei geeigneter Zusammenarbeit einen Wissenszuwachs erfahren könnte) (Epstein 1995, Horne et al. 1999). Hier liegt insofern ein Verbesserungspotenzial vor, dass im Entlassungsbrief auf Medikamentenänderungen der Krankenhausärzte hingewiesen werden sollte. Möglich wäre auch eine detaillierte, eventuell telefonische Information des Hausarztes durch den Klinikarzt bezüglich möglicher Fragen zur Entlassungsmedikation. Somit können Änderungen der Verordnung in einem überschaubaren Rahmen gehalten werden. Vor diesem Hintergrund startete 2005 an der Abteilung Allgemeinmedizin der Universität Heidelberg das Projekt „HeiCare“. In diesem Modell sendet der Hausarzt vor der Einweisung des Patienten eine Arzneimittel-Anamnese an die Klinik, die die medikamentöse Therapie mit der hausinternen Apotheke, der hausärztlichen Medikation und den momentanen Bedürfnissen des Patienten abstimmt. Der Hausarzt erhält noch vor der Entlassung eine Vorabinformation und anschließend einen detaillierten Arztbrief. Der Patient bekommt ein Informationspapier durch die Klinik und ein ergänzendes Beratungsgespräch durch den Hausarzt (www.klinikum.uni-heidelberg.de 2006). 


\section{Fragestellung und Ziel}

Die vorliegende Arbeit sollte die Umsetzung wesentlicher evidenzbasierter Kriterien bei Patienten mit Herzinsuffizienz in der prästationären, stationären und poststationären Pharmakotherapie untersuchen. Dabei stand im Vordergrund, ob

- alle herzinsuffizienten Patienten einen ACE-Hemmer erhielten,

- Patienten, denen kein ACE-Hemmer verschrieben wurde, einen AT-II-Antagonisten erhielten,

- bevorzugt evidenzbasierte Betablocker (Metoprolol, Bisoprolol, Carvedilol) verordnet wurden,

- Aldosteronantagonisten bevorzugt bei den höheren Schweregraden der Herzinsuffizienz eingesetzt wurden.

Neben der medikamentösen Therapie sollten Strategien der nichtmedikamentösen Behandlung untersucht werden. Im Einzelnen sollte analysiert werden, ob Patienten mit Herzinsuffizienz

- sich kochsalzarm ernährten,

- ihre tägliche Trinkmenge kontrollierten,

- ihr Gewicht kontrollierten und im Falle einer Gewichtszunahme entsprechend reagierten (Diuretikaerhöhung, Arztbesuch),

- sich regelmäßig gegen Grippe und/oder Pneumokokken impfen ließen.

Ein weiteres Ziel dieser Arbeit war die Analyse der Kontinuität der Therapie an der ambulant-stationären Schnittstelle (und umgekehrt). Analysenschwerpunkt war die Kontinuität der medikamentösen Verordnungsraten zwei Wochen nach Entlassung. 


\section{Material und Methoden}

Die Patientenrekrutierung erfolgte von November 2002 bis Dezember 2003 auf den internistisch-kardiologischen Stationen des Universitätsklinikums Göttingen. Die aktive Rekrutierung der Patienten erfolgte während des Semesters und erstreckte sich somit in oben genanntem Zeitraum über neun Monate. Patienten mit behandlungsbedürftiger Herzinsuffizienz wurden mit Hilfe des Stationsarztes, der Patientenakten und des Einweisungsscheins identifiziert.

Die Rekrutierung der Patienten erfolgte durch zwei Personen, ebenso die telefonische Befragung 14 Tage nach Entlassung (siehe Anhang), um somit die Beeinflussung der Ergebnisse durch den Befragungsstil möglichst gering zu halten.

\subsection{Patienten}

Folgende Einschluss- bzw. Ausschlusskriterien wurden zur Rekrutierung der Patienten herangezogen:

\section{Einschlusskriterien:}

- Diagnose Herzinsuffizienz jeden NYHA-Stadiums

- Ejektionsfraktion im Echokardiogramm $\leq 45 \%$

- Mindestalter 18 Jahre

- ausreichende Kommunikationsfähigkeit (z.B. Deutschkenntnisse)

- schriftliches Einverständnis zur Teilnahme an der Studie.

\section{Ausschlusskriterien:}

- unzureichende Kommunikationsfähigkeit (mangelnde Deutschkenntnisse, Aphasien)

- fehlende Einwilligung, bzw. Einwilligungsfähigkeit

- schwerwiegende Erkrankungen (z.B. Neoplasien, terminale Niereninsuffizienz)

- Patienten, die zur Weiterverlegung vorgesehen waren und nicht absehbar in die ambulante Betreuung entlassen werden konnten. 


\subsection{Datenerhebung}

Von 1785 Patienten, die sich während der 14-monatigen Laufzeit der Studie auf den internistisch-kardiologischen Stationen befanden, konnten im aktiven Rekrutierungszeitraum 235 Patienten mit Herzinsuffizienz identifiziert werden. Einen Überblick über die Zusammensetzung unseres Patientenkollektivs gibt Abbildung 1.

91 Patienten, die ihre schriftliche Einwilligung gaben und gesundheitlich in der Lage dazu waren, wurden während des Klinikaufenthaltes anhand eines standardisierten Dokumentationsbogens (siehe Anhang) zu Begleiterkrankungen und Medikamenteneinnahme (hausärztliche Verordnung) befragt. Außerdem wurden anamnestische und verhaltensbezogene Daten (kochsalzarme Ernährung, Kontrolle der Trinkmenge, Wiegen, Hinzuziehen eines Arztes bei Gewichtszunahme, selbstständiges Anpassen der Diuretikadosis, Impfverhalten) erhoben.

Die Angaben über die prästationäre Medikation des Hausarztes wurden anschließend durch Informationen aus dem Aufnahmegespräch und dem Einweisungsschein überprüft. Danach wurden der Schweregrad der Herzinsuffizienz nach NYHA, die kardiale Grunderkrankung, die Ejektionsfraktion und die geplante diagnostische oder therapeutische Intervention durch Akteneinsicht (Chart Review) ermittelt. Als weitere Informationsquelle stand der Entlassungsbrief zur Verfügung, dem die Krankhausmedikation entnommen wurde.

14 Tage nach der Entlassung wurde jeder Patient telefonisch nach der aktuellen Medikation befragt. Auch hier wurden Fragen zur nicht-medikamentöse Therapie der Herzinsuffizienz und zum Krankheitsverhalten gestellt. Außerdem ermittelten wir das NYHAStadium. Gleichzeitig erhielt der zuständige Hausarzt per Telefax ein Formblatt, auf dem er seine hausärztliche Medikation vor und nach Krankenhausaufenthalt eintrug. Die Faxantworten wurden dazu genutzt, im Falle fehlender Patientenangaben die Daten zu ergänzen.

Die entsprechenden Erhebungsbogen finden sich im Anhang. Allerdings sind nicht alle erhobenen Daten Gegenstand dieser Arbeit. 


\section{Abbildung 1: Ablaufdiagramm zur Studienteilnahme}

\section{Gesamtpatientenzahl internistisch-kardiologische Stationen November 2002 - Dezember 2003}

1785

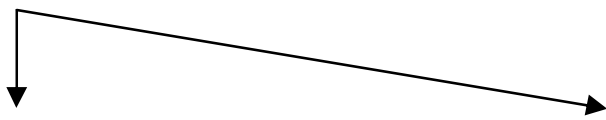

Patienten mit

Patienten ohne Herzinsuffizienz Herzinsuffizienz

367 1418

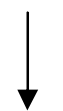

Patienten mit Herzinsuffizienz im aktiven Rekrutierungszeitraum (9 Monate) 235

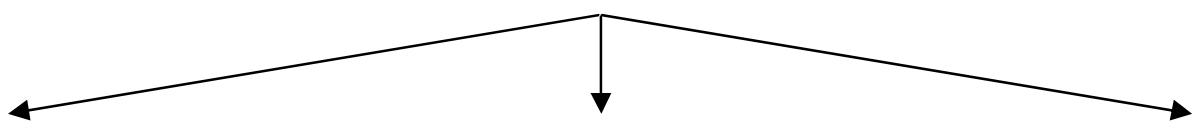

nachträglich
ausgeschlossene
Patienten*

53

$$
\begin{aligned}
& \text { den Einschlusskriterien } \\
& \text { entsprechende Patienten }
\end{aligned}
$$

151 nicht

erfasste

Patienten ${ }^{\star *}$

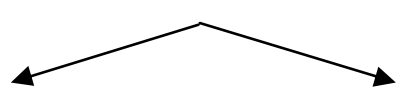

31

\section{Männer} 105

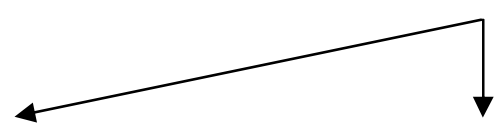

die Teilnahme abgelehnt 28 der Teilnahme zugestimmt 77
Frauen 46

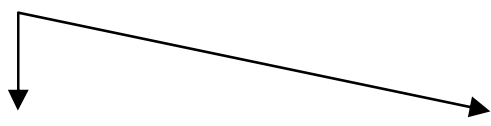

der Teilnahme zugestimmt 14 die Teilnahme abgelehnt 32

* Die Patienten wurden nachträglich ausgeschlossen wegen: Ejektionsfraktion > 45\%, nicht ausgefülltem Baseline-Fragebogen, zurückgezogener Bereitschaft zur Studienteilnahme.

** Diese Patienten konnten aufgrund von zu kurzem Aufenthalt auf Station (zum Beispiel im Rahmen von umgehenden Weiterverlegungen) oder Abwesenheit auf Station (unterwegs zu Diagnostik und Intervention) nicht rekrutiert werden. 


\subsection{Dateneingabe}

Die Dateneingabe erfolgte in anonymisierter Form in eine Access-Datenbank, die in eine SPSS-Datei umgewandelt wurde. Die weiteren Auswertungen erfolgten mit dem Statistikprogramm SPSS (Version 12.0). Zur statistischen Analyse kamen der CochranQ-Test und der Chi-Quadrat-Test zur Anwendung.

Alle verordneten Medikamente wurden nach der Anatomisch-therapeutischen-chemischen Klassifikation für den deutschen Arzneimittelmarkt (ATC-Code, Stand Mai 2003) codiert, um eine Einteilung in Medikamentengruppen sowie die statistische Auswertung zu ermöglichen. Die Codierung der Begleiterkrankungen wurde mit der ICD-10, Version 3.0, Stand Januar 2000 (DIMDI 1999) vorgenommen.

\subsection{Datenschutz und Genehmigung durch die Ethik-Kommission}

Die Studie zur Erfassung der Qualität der poststationären Versorgung wurde am 03.09.02 im Rahmen des Ethik-Antrages (Aktennummer: 30/7/02) der MedViP-Studie genehmigt und durchgeführt.

Eine Patientenbefragung fand nur statt, wenn eine schriftliche Einwilligung nach vorheriger vollständiger Aufklärung vorlag (informed consent). Sowohl die Patientenfrage- als auch die Dokumentationsbogen wurden unter Verschluss gehalten. Die elektronische Speicherung von Patientendaten und Untersuchungsergebnissen erfolgte in anonymisierter Form und ohne personenbezogene Angaben. Die erhobenen Daten wurden nicht an Dritte weitergegeben. 


\section{Ergebnisse}

Von November 2002 bis Dezember 2003 wurden 151 Patienten kontaktiert. 91 Patienten konnten in die Studie eingeschlossen werden (77 Männer, 14 Frauen). 32 Frauen lehnten eine Studienteilnahme aus Ängstlichkeit bzw. Skepsis ab. Bei 14 Patienten war bei der letzten Kontaktierung 14 Tage nach Entlassung aus der Klinik keine Angabe zur Medikation zu erhalten. Die an die Hausärzte gesandten Faxvorlagen erhielten wir in 55 Fällen ausgefüllt zurück.

\subsection{Patientencharakteristika}

\subsubsection{Geschlechterverteilung, Alter, Aufenthaltsdauer}

Der Anteil der männlichen Patienten überwog mit 77 (84,6\%) im Patientenkollektiv (91 Patienten) deutlich. Das durchschnittliche Patientenalter lag bei 67,9 Jahren. Die männlichen Patienten waren im Durchschnitt 66,8 Jahre (Range: 38 bis 84), die weiblichen Patienten 73,8 Jahre (Range: 65 bis 82), 8 Patienten (8,8\%) waren jünger als 50 Jahre. 28,6\% der Patienten waren jünger als 65 Jahre. Die durchschnittliche Dauer des stationären Aufenthalts betrug 19,44 Tage und umfasste eine Spanne von 2 bis 74 Tagen.

\subsubsection{NYHA - Stadien}

Diagramm 1a zeigt die Zugehörigkeit zu den NYHA-Stadien bei Rekrutierung. Am stärksten vertreten war das NYHA-Stadium III mit insgesamt 37 Patienten (40,7\%). Das durchschnittliche Patientenalter stieg von Stadium I bis Stadium IV an. Verglichen mit dem Rekrutierungszeitpunkt wiesen die Patienten 14 Tage nach Entlassung deutlich niedrigere Schweregrade der Herzinsuffizienz auf (Diagramm 1b). 


\section{Diagramm 1a: NYHA-Stadien bei Rekrutierung}

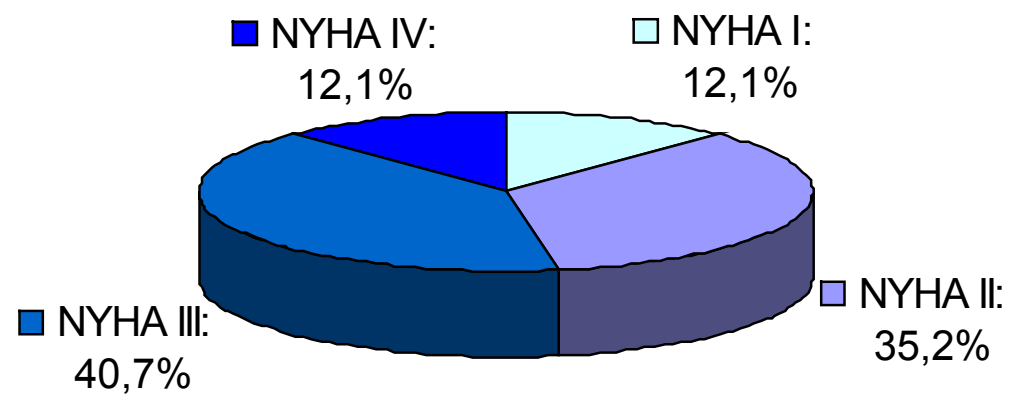

\section{Diagramm 1b: NYHA-Stadien 14 Tage nach Entlassung}

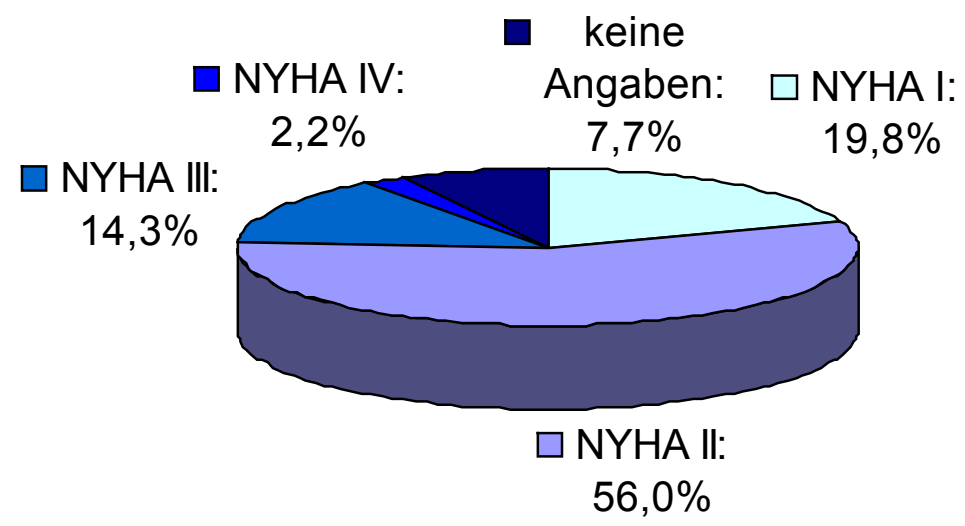

\subsubsection{Ejektionsfraktion}

Diagramm 2 zeigt die Verteilung der Ejektionsfraktion für die einzelnen NYHA-Gruppen. Der Mittelwert der Ejektionsfraktion lag bei 31,2\%. Die Patienten im Stadium NYHA I wiesen mit 35\% die höchste Ejektionsfraktion des beobachteten Kollektivs auf. 


\section{Diagramm 2: Ejektionsfraktion - NYHA-Stadium}

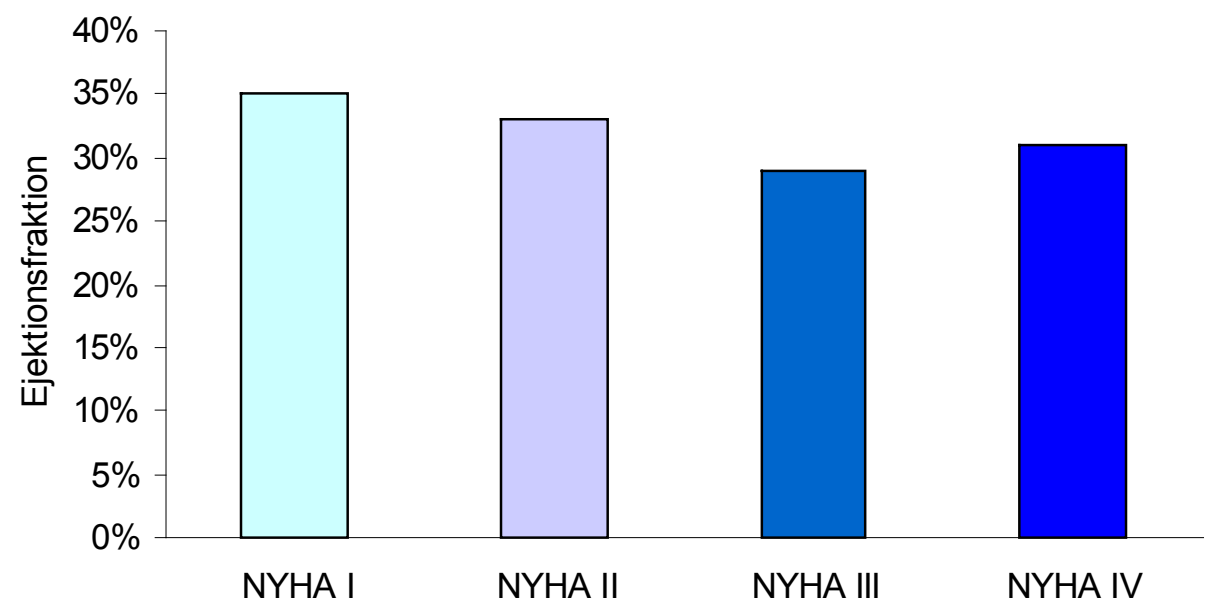

\subsubsection{Grunderkrankungen und diagnostisch-therapeutische Maßnahmen}

Weiterhin untersuchten wir in unserem Patientenkollektiv die der Herzinsuffizienz zugrunde liegenden Erkrankungen. Bei 53,8\% der Patienten lag eine koronare Herzkrankheit und bei 58,2\% ein Hypertonus vor. Die dilatative Kardiomyopathie (DCM) sowie die ischämische Kardiomyopathie (ICM) traten mit 19,8\% bzw. mit 22,0\% deutlich seltener auf. Herzvitien (z.B. Mitralinsuffizienz) kamen in 20,9\% der Fälle vor. Eine absolute Arrhythmie bestand in 33,0\% der Fälle.

Von den 91 beobachteten Patienten erhielten 51 eine invasive diagnostischtherapeutische Maßnahme. Eine Herzkatheteruntersuchung wurde bei 45 Patienten $(49,5 \%)$ durchgeführt, eine perkutane transluminale Koronarangioplastie (PTCA) mit Stenteinlage bei 9 Patienten (9,9\%). 11 Patienten (12,1\%) erhielten während ihres Klinikaufenthaltes einen Schrittmacher. Größere Eingriffe, wie eine koronare Bypass-OP bzw. ein Klappenersatz, erfolgten in je 2,2\% der Fälle $(n=2)$. 


\subsection{Pharmakotherapie der Herzinsuffizienz}

Der Blick auf die Gesamtverordnungszahl aller Stoffklassen zeigt, dass im Krankenhaus signifikant mehr Medikamente verschrieben wurden als ambulant. Dies ist insofern von Bedeutung, als sich dieser Trend auch bei der Betrachtung der einzelnen Stoffklassen fortsetzte: An der Schnittstelle zur Krankenhauseinweisung nahm die Verschreibungszahl zu; 14 Tage nach Entlassung fiel sie leicht ab, lag aber immer noch über dem Ausgangsniveau. 
Tabelle 2: Prästationäre, stationäre und poststationäre Pharmakotherapie der Herzinsuffizienz

\begin{tabular}{|c|c|c|c|c|}
\hline Medikamentengruppen & $\begin{array}{l}\text { Vor Aufnahme } \\
\mathrm{n}=91\end{array}$ & $\begin{array}{l}\text { Stationär } \\
\mathrm{n}=91\end{array}$ & $\begin{array}{l}\text { Nach Entlassung } \\
\mathrm{n}=77^{*}\end{array}$ & Statistik \\
\hline Gesamtzahl der Verordnungen & $6,72 \pm 3,20$ & $8,53 \pm 2,79$ & $7,05 \pm 7,00$ & $\begin{array}{l}\chi^{2}[2]=22,62 ; W=0,15 \\
p=0,001\end{array}$ \\
\hline Thiazid- und Schleifendiuretika & $70,3 \%(64)$ & $85,7 \%(78)$ & $81,0 \%(64)$ & $Q[2]=16,33 ; p=0,001$ \\
\hline ACE-Hemmer & $54,9 \%(50)$ & $72,5 \%(66)$ & $71,4 \%(55)$ & $Q[2]=14,86 ; p=0,001$ \\
\hline Betablocker gesamt & $64,3 \%(59)$ & $89,0 \%(81)$ & $85,7 \%(66)$ & $Q[2]=17,58 ; p=0,001$ \\
\hline $\begin{array}{l}\text { - Evidenzbasierte Betablocker: (Metoprolol } \\
\text { oder Bisoprolol oder Carvedilol) }\end{array}$ & $56,0 \%(51)$ & $82,4 \%(75)$ & $77,9 \%(60)$ & $Q[2]=20,67 ; p=0,001$ \\
\hline - Metoprolol & $27,5 \%(25)$ & $44,0 \%(40)$ & $39,0 \%(30)$ & $Q[2]=10,78 ; p=0,005$ \\
\hline - Bisoprolol & $15,4 \%(14)$ & $25,3 \%(23)$ & $26,0 \%(20$ & $\mathrm{Q}[2]=7,60 ; p=0,022$ \\
\hline - Carvedilol & $13,2(12)$ & $13,2 \%(12)$ & $13,0 \%(10)$ & $Q[2]=0,25 ; p=0,882 ;$ n.s. \\
\hline Digitalisglykoside & $31,9 \%(29)$ & $41,8 \%(38)$ & $41,6 \%(32)$ & $Q[2]=6,64 ; p=0,036$ \\
\hline AT-II-Antagonisten & $13,2 \%(12)$ & $11,0 \%(10)$ & $14,3 \%(11)$ & $Q[2]=2,00 ; p=0,368 ;$ n.s. \\
\hline Kaliumsparende Diuretika & $3,3 \%(3)$ & $3,3 \%(3)$ & $5,2 \%(4)$ & $Q[2]=0,40 ; p=0,819 ;$ n.s. \\
\hline Aldosteronantagonisten & $16,5 \%(15)$ & $38,5 \%(35)$ & $33,8 \%(26)$ & $Q[2]=21,58 ; p=0,001$ \\
\hline
\end{tabular}

$\mathrm{Q}=$ Cochrans $\mathrm{Q}-$ Test $; \mathrm{W}=$ Kendall-W-Koeffizient 


\subsubsection{Verordnung von ACE-Hemmern}

In der Klinik werden signifikant häufiger ACE-Hemmer verordnet. Am Übergang zur poststationären Therapie wird in neun Fällen der ACE-Hemmer wieder abgesetzt. Diagramm 3 zeigt, dass sich der Großteil der Neuanordnungen im Krankenhaus auf Patienten im Stadium NYHA I (11 Patienten) konzentriert. Diese Beobachtung wäre im Rahmen einer größeren Studie mit höheren Patientenzahlen zu prüfen.

Diagramm 3: Verordnung von ACE-Hemmern in Abhängigkeit vom Schweregrad nach NYHA

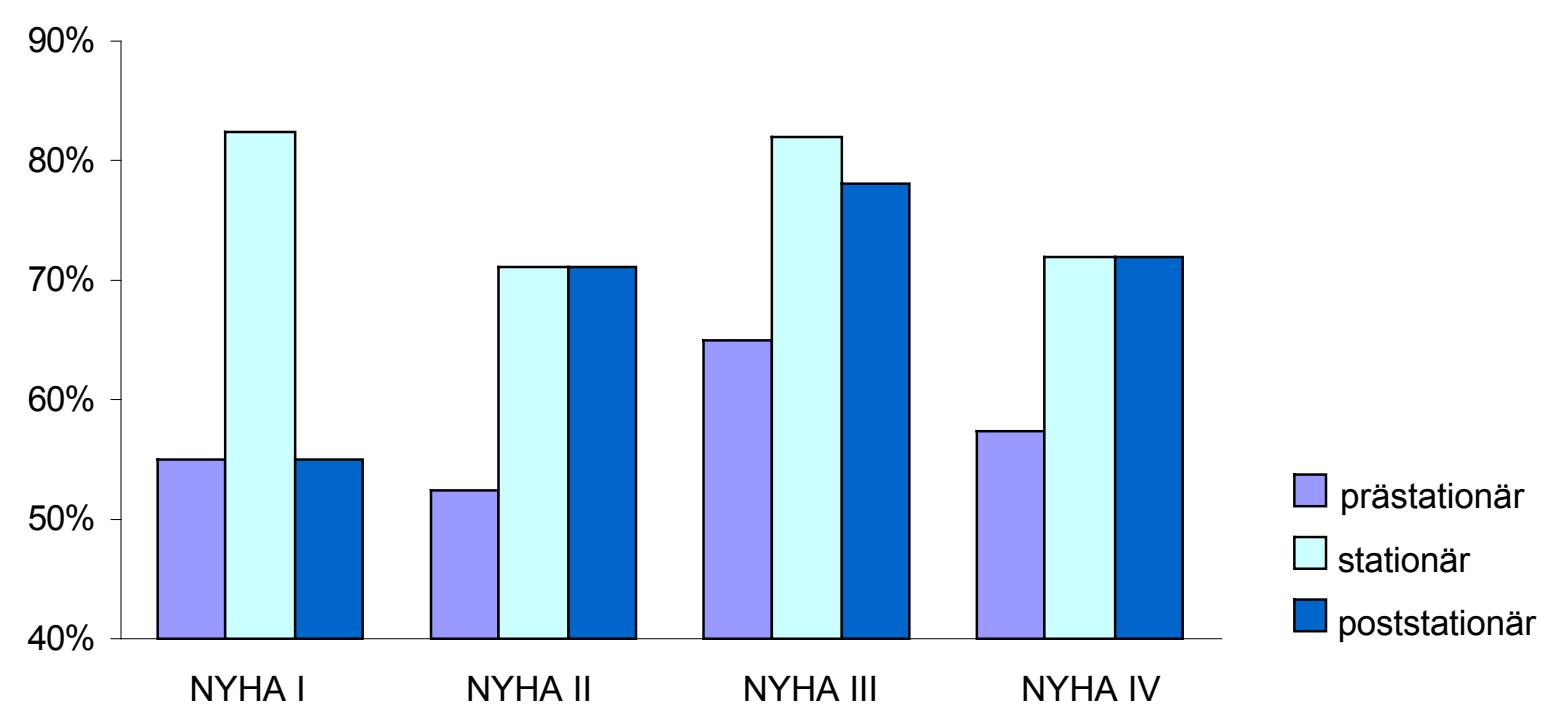

\subsubsection{Verordnung von AT-II-Antagonisten}

Insgesamt wurden AT-II-Antagonisten zu allen drei Beobachtungszeitpunkten zurückhaltend verschrieben. Zwischen prästationärer, stationärer und poststationärer Medikation ergeben sich keine signifikanten Unterschiede. Von den Patienten, die keinen ACEHemmer erhielten, bekamen vor Einweisung 29,3\% (12 von 41), im Krankenhaus 40,0\% (10 von 25) und 14 Tage nach Entlassung 50\% (11 von 22) einen AT-IIAntagonisten. Die Rate der verordneten AT-II-Antagonisten ist somit stabil und fällt poststationär prozentual besser aus als stationär (nicht signifikant). 


\subsubsection{Verordnung von evidenzbasierten Betablockern}

Betablocker wurden im Krankenhaus signifikant häufiger verschrieben als prästationär. 14 Tage nach Entlassung zeigt sich dieser Effekt nur leicht abgeschwächt. In der stationären Therapie gehörten 92,6\% aller verordneten Betablocker der Gruppe der prognoseverbessernden Betablocker an. Vor der Einweisung machten Metoprolol, Bisoprolol oder Carvedilol nur $86,4 \%$, poststationär immerhin noch $90,9 \%$ der verordneten Betablocker aus. In der stationären Pharmakotherapie werden signifikant häufiger evidenzbasierte Betablocker ausgewählt als ambulant.

\subsubsection{Verordnung von Aldosteronantagonisten}

Wie bei den anderen Medikamentengruppen wurden auch die Aldosteronantagonisten im Krankenhaus signifikant häufiger verschrieben als prästationär (siehe Tabelle 2). Allerdings konzentrieren sich die Mehrverschreibungen (anders als nach evidenzbasierten Kriterien zu erwarten) nicht auf die höheren Schweregrade der Herzinsuffizienz. Vielmehr verteilen sie sich relativ gleichmäßig auf alle NYHA-Gruppen und sind NYHA I und II $(n=43)$ sogar noch etwas häufiger zuzuordnen als NYHA III und IV $(n=48)$, wie Diagramm 4 veranschaulicht. Zu allen drei Beobachtungszeitpunkten sind die Empfehlungen bezüglich eines bevorzugten Einsatzes der Aldosteronantagonisten bei schwerer Erkrankten somit nicht umgesetzt. 
Diagramm 4: Verordnung von Aldosteronantagonisten in Abhängigkeit vom Schweregrad nach NYHA

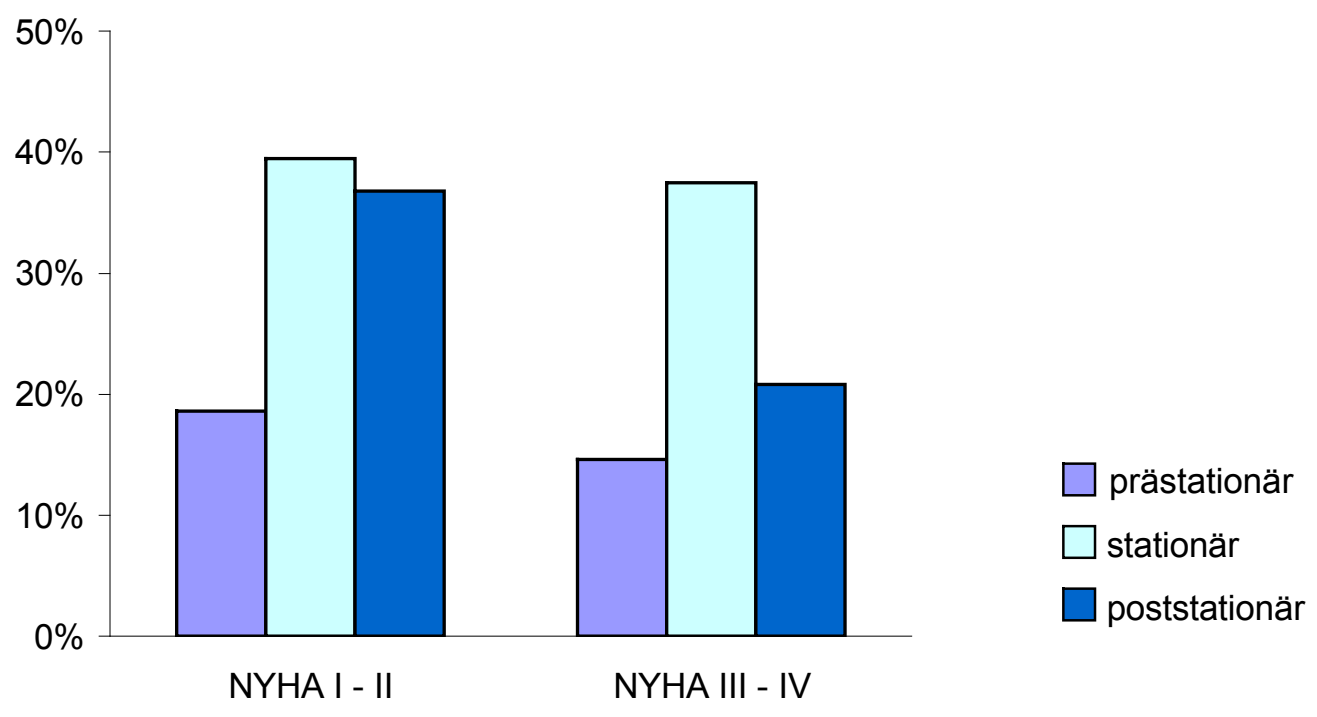

\subsubsection{Medikamentenwechsel an der ambulant-stationären Schnittstelle}

\section{Schnittstelle Hausarzt/Krankenhaus}

Insgesamt wurden nur 36,9\% der hausärztlichen Herzinsuffizienzmedikamente ohne Änderung des Wirkstoffs übernommen. Neben der hohen Zahl von Neuverordnungen wurden in der stationären Therapie auch Wirkstoffwechsel innerhalb der einzelnen Stoffgruppen vorgenommen. Die häufigsten Wirkstoffwechsel finden sich in der Gruppe der Thiazid- und Schleifendiuretika. Hier wurden 42,2\% der hausärztlich verordneten Substanzen durch ein Analogon ersetzt. Bei ACE-Hemmern wurde in 36,0\%, bei Betablockern in $23,7 \%$ der Fälle der Wirkstoff ersetzt. 
Tabelle 3: Medikamentenwechsel im Krankenhaus

\begin{tabular}{|c|c|c|c|c|}
\hline \multirow{2}{*}{ Medikamentengruppen } & \multicolumn{3}{|c|}{$\begin{array}{l}\text { Hausärztliche Medikation } \\
\text { durch das Krankenhaus }\end{array}$} & \multirow{2}{*}{$\begin{array}{c}\text { Im Kranken- } \\
\text { haus neu } \\
\text { angesetzt } \\
(\%)\end{array}$} \\
\hline & abgesetzt (\%) & $\begin{array}{l}\text { ersetzt durch } \\
\text { Analogon (\%) }\end{array}$ & $\begin{array}{c}\text { beibehalten } \\
(\%)\end{array}$ & \\
\hline ACE-Hemmer & 5,7 & 25,7 & 40,0 & 28,6 \\
\hline Betablocker & 3,6 & 16,7 & 50,0 & 29,8 \\
\hline AT-II-Antagonisten & 28,6 & 14,3 & 42,9 & 14,3 \\
\hline Aldosteronantagonisten & 7,9 & 5,3 & 26,3 & 60,5 \\
\hline Digitalisglykoside & 13,6 & 4,5 & 47,7 & 34,1 \\
\hline $\begin{array}{l}\text { Thiazid- und } \\
\text { Schleifendiuretika }\end{array}$ & 1,3 & 34,2 & 31,6 & 32,9 \\
\hline $\begin{array}{l}\text { Kaliumsparende } \\
\text { Diuretika }\end{array}$ & 40,0 & 0,0 & 20,0 & 40,0 \\
\hline
\end{tabular}

\subsubsection{Medikamentenwechsel an der stationär-ambulanten Schnittstelle}

Schnittstelle Krankenhaus/Hausarzt

Zwei Wochen nach Entlassung wurden 67,9\% der klinischen Herzinsuffizienzmedikation ohne Änderung des Wirkstoffs übernommen. $88,4 \%$ der Betablocker wurden, ohne eine Wirkstoffänderung vorzunehmen, durch den Hausarzt weiterverordnet und sind somit die Medikamentengruppe mit der größten Verordnungskonstanz, dicht gefolgt von den Digitalisglykosiden mit 87,1\% und den ACE-Hemmern mit 76,2\%. Entsprechend seltener wurden Medikamente ersetzt oder neu angesetzt, mit Ausnahme der AT-IIAntagonisten. Diese wurden in $23,1 \%$ der Fälle durch den Hausarzt neu angesetzt und 
in nur $15,4 \%$ der Fälle wurde ein im Krankenhaus verordneter AT-II-Antagonist durch den Hausarzt abgesetzt.

Tabelle 4: Medikamentenwechsel 14 Tage poststationär

\begin{tabular}{|c|c|c|c|c|}
\hline \multirow{2}{*}{ Medikamentengruppen } & \multicolumn{3}{|c|}{$\begin{array}{l}\text { Krankenhausmedikation } \\
\text { durch den Hausarzt }\end{array}$} & \multirow{2}{*}{$\begin{array}{l}\text { Durch den } \\
\text { Hausarzt neu } \\
\text { angesetzt } \\
(\%)\end{array}$} \\
\hline & abgesetzt (\%) & $\begin{array}{l}\text { ersetzt durch } \\
\text { Analogon (\%) }\end{array}$ & $\begin{array}{c}\text { beibehalten } \\
(\%)\end{array}$ & \\
\hline ACE-Hemmer & 9,5 & 11,1 & 76,2 & 3,2 \\
\hline Betablocker & 5,7 & 4,3 & 87,1 & 2,9 \\
\hline AT-II-Antagonisten & 15,4 & 7,7 & 53,8 & 23,1 \\
\hline Aldosteronantagonisten & 16,1 & 12,9 & 67,7 & 3,2 \\
\hline Digitalisglykoside & 17,5 & 2,5 & 75,0 & 5,0 \\
\hline $\begin{array}{l}\text { Thiazid- und } \\
\text { Schleifendiuretika }\end{array}$ & 4,5 & 11,9 & 76,1 & 7,5 \\
\hline $\begin{array}{l}\text { Kaliumsparende } \\
\text { Diuretika }\end{array}$ & 20,0 & 0,0 & 40,0 & 40,0 \\
\hline
\end{tabular}

\subsection{Nichtmedikamentöse Therapie der Herzinsuffizienz}

Kochsalzarme Ernährung:

39,1\% der befragten Patienten gaben an, sich kochsalzarm zu ernähren. Die Mehrheit der Patienten (56,5\%) verneinte eine kochsalzrestriktive Ernährung. 


\section{Kontrolle der Trinkmenge:}

Zur Kontrolle der täglichen Trinkmenge befragt, gaben 66,9\% der Patienten an, diese nicht zu kontrollieren. 28,7\% der Patienten beschränkten ihre tägliche Trinkmenge.

\section{Wiegen und Umgang mit Gewichtszunahme:}

24 Patienten $(26,4 \%)$ gaben an, sich nie oder seltener als einmal pro Monat zu wiegen. Neun Patienten (9,9\%) wiegen sich ca. einmal im Monat. Weitere 15 Patienten (16,5\%) wiegen sich ca. einmal pro Woche und die Mehrzahl der Befragten (39 Patienten, bzw. 42,9\%) kontrolliert ihr Gewicht mehrmals wöchentlich bis täglich.

Weiterhin wurden die Patienten nach ihrem Verhalten befragt, falls sie innerhalb von zwei Tagen 2-3 kg an Gewicht zunehmen sollten. 25,3\% würden unter diesen Umständen einen Arzt aufsuchen oder kontaktieren, 5 von 91 Patienten (5,5\%) würden eigenständig ihre Diuretikadosis anpassen.

\section{Impfverhalten:}

Diagramm 5 und 6 veranschaulichen die Angaben zum Impfverhalten unserer Stichprobe bezüglich der Grippe- und der Pneumokokkenimpfung.

\section{Diagramm 5: Grippeimpfung}

口 unbekannt:

$4,4 \%$

$\square$ nein: $41,6 \%$

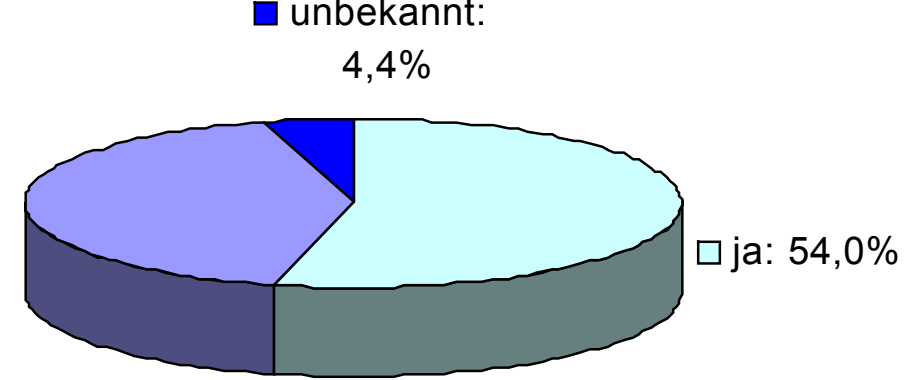




\section{Diagramm 6: Pneumokokkenimpfung}

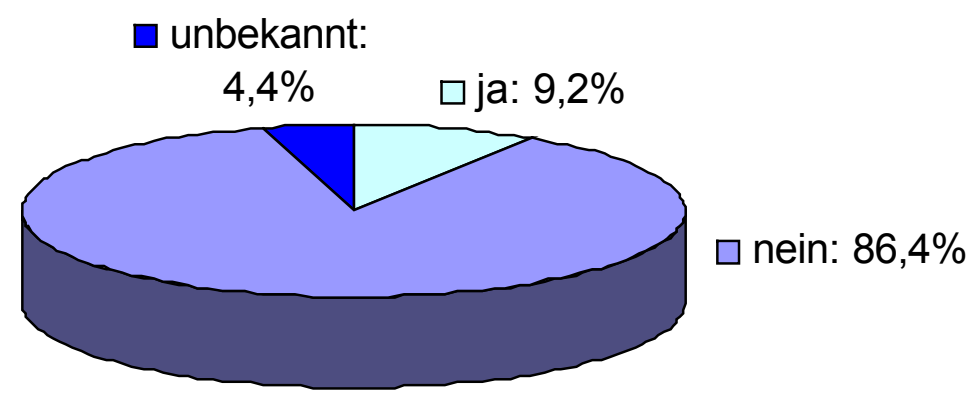

54\% der Patienten gaben an, sich jährlich gegen Grippe impfen zu lassen. Nur 9,2\% der Patienten gaben an, sich alle fünf Jahre gegen eine Pneumokokkeninfektion impfen zu lassen. Vier Patienten konnten zu Impfungen keine Aussage machen. 


\section{Diskussion}

Die vorliegende Arbeit untersucht die Umsetzung evidenzbasierter Therapiekriterien bei der chronischen Herzinsuffizienz im prästationären, stationären und poststationären Vergleich. Für das Krankenhaus konnte, mit Ausnahme der Aldosteronantagonisten (siehe unten), eine konsequentere Umsetzung der aktuellen Therapieempfehlungen gegenüber hausärztlichen Kollegen beobachtet werden. Dies spiegelt sich in höheren Verordnungszahlen bei ACE-Hemmern und evidenzbasierten Beta-Blockern (MetoproIol, Bisoprolol und Carvedilol) in der Klinik wider. Auch die Aldosteronantagonisten werden im Krankenhaus häufiger eingesetzt, doch nicht (wie nach den Empfehlungen der Leitlinien zu erwarten) bei den höheren NYHA-Stadien. In unserer Studie zeigten sich höhere Verordnungsraten in den NYHA-Stadien I und II. In den NYHA-Stadien III und IV sinkt die Verordnungshäufigkeit ab. Insgesamt wurden bei Aufnahme in die Klinik nur $36,9 \%$ der hausärztlichen Medikamente weiterverordnet. 14 Tage nach Entlassung wurden immerhin $67,9 \%$ der Kliniksmedikation durch den Hausarzt beibehalten. Bezüglich der berichteten Umsetzung nichtmedikamentöser Therapieempfehlungen zeigten sich Defizite bei der kochsalzarmen Ernährung, der Kontrolle der Trinkmenge, dem Wiegen und dem Umgang mit Gewichtszunahme sowie beim Impfverhalten.

\subsection{Limitationen}

Unsere Stichprobe ist bezüglich des Geschlechts asymmetrisch verteilt: 84,6\% der 91 rekrutierten Patienten sind Männer, 15,4\% sind Frauen. Eine geschlechtsspezifische Differenzierbarkeit unserer Aussagen ist aufgrund des geringen Frauenanteils unserer Stichprobe somit nicht gegeben.

Weiterhin ist zu berücksichtigen, dass die Patientenrekrutierung am Universitätsklinikum Göttingen, einem Haus der Maximalversorgung, erfolgte. Die Daten bezüglich des stationären Aufenthaltes eignen sich somit nicht zur Verallgemeinerung auf Kliniken insgesamt. Der krankenhausbezogene Ansatz bietet hingegen den Vorteil, dass ein breites Spektrum von Hausärzten auch aus dem weiteren Göttinger Umfeld und deren Verordnungen erreichbar und die getroffenen Aussagen eher generalisierbar sind. 
Die in dieser Arbeit getroffenen Aussagen basieren auf der statistischen Analyse von Verschreibungshäufigkeiten kardial wirksamer Medikamente. Wie leitlinienadhärent bzw. medizinisch indiziert die hausärztliche Verordnung im Einzelnen ist, kann letztlich nur unter Berücksichtigung jeder individuellen Anamnese erfolgen und erlaubt keine Bewertung der Behandlungsqualität im Einzelfall.

\section{$6.2 \quad$ Kontext}

Die im Folgenden detaillierter diskutierten Daten unserer Studie fügen sich in die internationale Studienlage zur Herzinsuffizienztherapie ein. Sie bilden die momentane medikamentöse Versorgungssituation herzinsuffizienter Patienten sowie die Umsetzung von Therapieempfehlungen für Deutschland im ambulanten und stationären Bereich am Beispiel des Göttinger Raumes ab und sind Grundlage der Weiterbeobachtung und Therapiedarstellung im Verlauf.

\section{Verordnung von ACE-Hemmern:}

Erwartungsgemäß erhielten nicht alle Patienten einen ACE-Hemmer. In der Klinik wurden jedoch signifikant häufiger ACE-Hemmer verordnet. 72,5\% der Patienten erhielten zum Entlassungszeitpunkt einen ACE-Hemmer. Dies entspricht den Ergebnissen vorliegender Studien. Eine amerikanische Studie, die an Universitätskliniken durchgeführt wurde, ergab Verordnungsraten von $72 \%$ für ACE-Hemmer zum Zeitpunkt der Entlassung (Nohria et al. 1999). Im Rahmen des EuroHeart Failure Survey programme konnten für Europa durchschnittliche Verordnungszahlen von 61,8\% zum Entlassungszeitpunkt erhoben werden (Komajda et al. 2003).

Poststationär geht die Verordnung der ACE-Hemmer nur geringfügig auf $71,4 \%$ zurück und liegt somit deutlich über der prästationären Ausgangsverordnung von 54,9\%. Somit liegen unsere Verordnungsraten über denen einer niederländischen (Kasje et al. 2005) und einer englischen Studie (Gnani et al. 2004).

Wie im Ergebnisteil geschildert, finden ACE-Hemmer in allen NYHA-Stadien Einsatz, werden jedoch im ambulanten Bereich in den NYHA-Stadien III und IV häufiger eingesetzt. Für das Krankenhaus deutet sich ein gehäufter Einsatz von ACE-Hemmern im NYHA-Stadium I an. Dies ist möglicherweise auf eine (im Vergleich zur ambulanten 
Versorgung) erhöhte apparative Ausstattung zurückzuführen. Als weiterer Erklärungsansatz kommt eine stärkere Akzentuierung von prognoseverbessernden Kriterien im Krankenhaus in Betracht. Aufgrund der geringen Patientenzahl (11) im NYHA-Stadium I scheint die Untermauerung dieser Tendenz durch weitere Studien sinnvoll.

\section{Verordnung von AT-II-Antagonisten:}

AT-II-Antagonisten wurden insgesamt selten eingesetzt. Den Patienten, die prästationär keinen ACE-Hemmer erhalten hatten, wurde in circa einem Drittel der Fälle ein AT-IIAntagonist verordnet. Stationär erhielten $40 \%$ und nach Entlassung sogar $50 \%$ der Patienten ohne ACE-Hemmermedikation einen AT-II-Antagonisten. Anders als zu vermuten, scheinen Hausärzte nach stationärem Aufenthalt fehlende ACE-Hemmer konsequenter zu substituieren, als dies in der prästationären oder stationären Therapie der Fall ist. Dies kann aber auch auf Empfehlungen im Arztbrief zurückzuführen sein. Diese Daten ergänzen die internationale Literatur insofern, als sie durch ein von den aktuellen Studien differentes methodisches Vorgehen gewonnen wurden. In der vorliegenden Arbeit wurden die Verordnungsraten für ACE-Hemmer und AT-II-Antagonisten gemeinsam analysiert. In einer Schweizer Studie wurden Verordnungsraten von $65 \%$ für die Medikamentengruppe der ACE-Hemmer/AT-II-Antagonisten beschrieben (Muntwyler und Follath 2000). In einer amerikanischen Studie, die Verordnungsraten von $52 \%$ für ACE-Hemmer und 9\% für AT-II-Antagonisten ergab, wurden ACE-Hemmer und AT-IIAntagonisten getrennt betrachtet (Schmedtje et al. 2003). Wie viele Patienten, (die keinen ACE-Hemmer erhalten) einen AT-II-Antagonisten erhalten, wird nur in wenigen Publikationen berichtet. Masoudi et al. (2004) beschreiben, dass bei Patienten mit der Aufnahmediagnose „Herzinsuffizienz“, zum Entlassungszeitpunkt Verschreibungsraten von $68 \%$ bei den ACE-Hemmern erreicht wurden. $76 \%$ der Patienten erhielten entweder einen ACE-Hemmer oder einen AT-II-Antagonisten. Im Vergleich zu diesen Daten wurden bei unseren Patienten häufiger AT-II-Antagonisten eingesetzt, wenn keine ACEHemmermedikation vorlag. 


\section{Verordnung von Beta-Blockern:}

Für Europa konnten im Rahmen des EuroHeart Failure Survey programme zum Zeitpunkt der Entlassung aus der Klinik gezeigt werden, dass 36,9\% der Patienten einen Beta-Blocker erhielten (Komajda et al. 2003). Ambulant erhielten 34\% der Patienten einen Beta-Blocker (Cleland et al. 2002). Die Verordnungsraten werden in der vorliegenden Literatur nach wie vor als zu niedrig beschrieben.

Im Vergleich zur vorliegenden Literatur (Erdmann et al. 2001, Hjalmarson et al. 2000, Packer et al. 2002) erhielten die Patienten unserer Stichprobe sowohl stationär als auch ambulant verhältnismäßig häufig Beta-Blocker (siehe oben). Im Krankenhaus kam es zu einem Anstieg der Beta-Blockerverordnungen auf 89\%. Nach Entlassung erhielten noch 85,7\% der Patienten einen Beta-Blocker. Ein hoher Prozentsatz der eingesetzten BetaBlocker gehörte zu den prognoseverbessernden (Bisoprolol, Metoprolol und Carvedilol). Stationär wurden 92,95\% der verordneten Beta-Blocker aus dieser Gruppe gewählt, prästationär $87,1 \%$ und poststationär 90,9\%. Metoprolol wurde zu allen drei Zeitpunkten am häufigsten eingesetzt.

Die prognoseverbessernden Beta-Blocker werden in unserer Stichprobe bevorzugt eingesetzt. Ihr Einsatz erfolgt dabei stationär signifikant häufiger als prästationär. Zusammenfassend zeigt sich hinsichtlich der Beta-Blocker auch in unserem Kollektiv ein Potenzial zur fokussierten Auswahl von evidenzbasierten Wirkstoffen (Bisoprolol, Metoprolol und Carvedilol).

\section{Verordnung von Aldosteronantagonisten:}

In den letzten Jahren zeichnete sich für die Gruppe der Aldosteronantagonisten bereits ein deutlicher Anstieg der Verordnungszahlen ab. Trotz dieser Verbesserungen wird in der internationalen Literatur eine weitere Steigerung der Verordnungszahlen angeregt (Grigorian et al. 2005). Eine kanadische Studie zeigte eine Zunahme der Verordnungen für Spironolacton von 2,2\% in den Jahren 1997/1998 auf 18,7\% in den Jahren 2001/2002 (Cox et al. 2005). Entgegen publizierter Empfehlungen (Task Force for the Diagnosis and Treatment of Chronic Heart Failure, European Society of Cardiology 2001) konnten wir für unser Patientenkollektiv keinen bevorzugten Einsatz der Aldosteronantagonisten in den höheren NYHA-Stadien nachweisen. Vielmehr zeigte sich eine recht homogene Verordnung in allen NYHA-Stadien mit höheren Verordnungsraten in 
den Stadien I und II (siehe hierzu auch Diagramm 4). Die Empfehlungen zum bevorzugten Einsatz der Aldosteronantagonisten in höheren NYHA-Stadien sind in diesem Fall weder von ambulanten noch von stationären Behandlern umgesetzt.

\section{Verordnungsverhalten an den Schnittstellen:}

Das Verordnungsverhalten an der Schnittstelle Hausarzt/Krankenhaus unterscheidet sich deutlich vom dem an der Schnittstelle Krankenhaus/Hausarzt. An der Schnittstelle Hausarzt/Krankenhaus kommt es zu häufigen Medikamentenwechseln bei der kardialen Medikation. Es werden lediglich 36,9\% der hausärztlichen Medikation ohne Änderung des Wirkstoffs übernommen. Dies entspricht den Befunden aus vorliegenden Studien. So wurden in einer deutschen Studie an der Schnittstelle Hausarzt/Krankenhaus 47\% der Medikamente ohne Veränderung übernommen (Himmel et al. 1996).

Unsere Studie zeigt, dass 14 Tage nach Entlassung der Großteil der Medikamentenverordnungen durch den Hausarzt weitergeführt werden. So wurden $67,9 \%$ der in der Klinik verschriebenen Medikamente ohne Änderung des Wirkstoffs vom Hausarzt übernommen. Die höchste Verordnungskonstanz wiesen hierbei die Betablocker auf. 14 Tage nach Entlassung wurden durch den Hausarzt der Großteil der Medikamentenverordnungen weitergeführt. Somit zeigt sich in unserer Studie 14 Tage nach Entlassung keine Diskontinuität in der Weiterverordnung geeigneter Herzinsuffizienzmedikamente. Vergleichbare Ergebnisse zur poststationären Behandlungskontinuität ergab, die bereits genannte Studie von Himmel et al. (1996), in der nach Entlassung 66,3\% der Medikamente durch den Hausarzt ohne Änderung übernommen wurden. Bei Studien mit späteren Nachbeobachtungszeitpunkten konnten jedoch auf längere Sicht ein Absinken der Verordnungszahlen gezeigt werden. So ergab die Auckland Heart Failure Management Study ein Absinken des ACE-Hemmereinsatzes in der Kontrollgruppe von $89 \%$ zum Baseline-Zeitpunkt auf 73\% im 12-Monats-Follow-up (Doughty et al. 2002). 


\section{Nichtmedikamentöse Therapie:}

Wichtige Eckpfeiler der nichtmedikamentösen Therapie der chronischen Herzinsuffizienz, deren Umsetzung auch heute noch nicht in ausreichendem Maße erfolgt, sind: die kochsalzarme Ernährung, die Kontrolle der täglichen Trinkmenge, das Wiegen und der Umgang mit Gewichtszunahme.

Dies spiegeln unsere Ergebnisse wider. Nur 39,1\% der Patienten gaben an, sich kochsalzarm zu ernähren. $28,7 \%$ gaben an, ihre tägliche Trinkmenge bewusst zu beschränken. Eine amerikanische Studie zeigte einen besseren Kenntnisstand (75\%) bezüglich Diätmaßnahmen. $11 \%$ der Studienpatienten gaben an, über die Notwendigkeit der täglichen Gewichtskontrolle informiert zu sein (Nohria et al. 1999). Dem entgegen stehen 42,9\% unserer Studienpatienten, die angaben sich mehrmals wöchentlich, bis täglich zu wiegen. Jedoch würden sich auch nur 25,3\% unserer befragten Patienten im Falle einer Gewichtszunahme an einen Arzt wenden.

Die Impfraten (bezüglich der in den Leitlinien empfohlenen Impfungen [siehe Kapitel 2.2.1 Hausarzt]) bei Patienten mit kardiovaskulären Erkrankungen sind mit 32,7\% nach wie vor zu niedrig. Allerdings erzielt die Patientengruppe mit der chronischen Herzinsuffizienz im Vergleich zu Patienten, die einen Schlaganfall erlitten haben, bezüglich der Influenzaimpfung bessere Werte $(37,1 \%$ vs. $31,4 \%)$ (Ajani et al. 2005). In unserer Stichprobe gaben deutlich mehr Patienten (54\%) an, sich jährlich gegen Grippe impfen zu lassen. Wesentlich weniger bekannt war die Möglichkeit der Pneumokokkenimpfung. Nur 9,2\% unserer Patienten gaben an, diese Impfung erhalten zu haben.

Bezüglich der nichtmedikamentösen Herzinsuffizienztherapie fügen sich unsere Daten in die international vorliegende Literatur ein und unterstreichen die Notwendigkeit weiterer Bemühungen um eine angemessene Patienteninformation (durch Ärzte, Arzthelferinnen und Angehörige) - mit dem Ziel einer verbesserten Lebensqualität und einer Reduktion der Rehospitalisierungsraten. 


\subsection{Bedeutung für die Praxis}

Von den Hausärzten werden verschiedene Gründe (siehe Kapitel 2.2.1) genannt, die die Umsetzung der vorliegenden Leitlinien im hausärztlichen Alltag schwierig gestalten. Somit ist die Implementation neuer bzw. in der Praxis umsetzbarer Leitlinien auch weiterhin ein wichtiger Aspekt in der Qualitätssicherung. Die Leitlinientreue der primärärztlich tätigen Ärzte steht jedoch nicht allein im Mittelpunkt neuerer Publikationen, ob nun fachgruppenspezifisch oder erkrankungsbezogen (Mottur-Pilson et al. 2001, McColl et al. 1998). Es müssen weitere Wege gefunden werden, um die Lücke in der Kommunikation und Versorgung am Übergang von der Klinik zum Hausarzt zu minimieren. Nur so können die initiierten Therapien auch auf lange Sicht konsequent und Erfolg versprechend umgesetzt werden.

Unsere Ergebnisse zeigen ein leitliniengerechteres Verordnungsverhalten bezüglich der Herzinsuffizienzmedikation in der Klinik als in der hausärztlichen Versorgung. Der für alle NYHA-Stadien empfohlene Einsatz von ACE-Hemmern wird sowohl stationär als auch ambulant noch nicht ausreichend umgesetzt. Konkret zeigt sich für die Medikamentengruppe der ACE-Hemmer im Bereich der ambulanten Versorgung ein Potenzial zum verstärkten Einsatz, speziell in den NYHA-Stadien I und IV. Für die Gruppe der Aldosteronantagonisten ergibt sich aus den vorliegenden Daten die Notwendigkeit des gezielteren Einsatzes in den NYHA-Stadien III und IV. Bezüglich der nichtmedikamentösen Therapie spiegeln unsere Daten den großen vorhandenen Informationsbedarf der betroffenen Patienten wider.

Neben der Umsetzung der oben erwähnten Punkte können noch weitere Aspekte für die Durchführung einer optimierten Therapie hilfreich sein. Hierzu gehört ein zeitnah verfasster, ausführlicher Entlassungsbrief der Klinik an den Hausarzt, in dem detailliert auf Therapiewechsel eingegangen wird. Die Option, mit Kollegen in der Klinik oder niedergelassenen Kollegen telefonisch Rücksprache zu halten oder einen Hausbesuch in der Klinik durchzuführen, bietet Gelegenheit: 
- zu ausgedehnterem Erfahrungsaustausch

- zur Klärung Fall-bezogener Fragen

- zum Informationsgewinn bezüglich neuer medikamentöser/nicht-medikamentöser Therapieansätze.

Hilfreich kann auch die Erstellung einer gemeinsamen Medikamentenliste, in Zusammenarbeit der Klinik und Hausärzte, bezogen auf die Hauptkrankheitsbilder sein (Hakansson et al. 2001). Dies kann die Medikamentenwechsel an den Schnittstellen vermindern, die Kontinuität in der Verordnung und die Therapiekonstanz erhöhen. Zusätzlich kann eine Kostenreduktion bezüglich verordneter Medikamente erreicht werden (Tomson et al. 1994).

\subsection{Bedeutung für die weitere Forschung}

Obwohl Hausärzte überzeugt sind, dass evidenz-basierte Medizin die Patientenversorgung verbessert, erfolgt die Umsetzung vorliegender Leitlinien nur bedingt, wie auch unsere Daten belegen (Mc Coll et al. 1998). Gründe für das Nichtbefolgen der Leitlinien müssen daher noch genauer erforscht werden, um anschließend Leitlinien erarbeiten zu können, die für den Bereich der Allgemein- und Familienmedizin mit der Multidimensionalität ihrer zu lösenden Aufgaben die Umsetzung evidenz-basierter Medizin ermöglichen bzw. vereinfachen. Weiterhin bietet es sich an, das Potenzial für gemeinsame/alternative (siehe Kapitel 6.3) Arbeitsbündnisse zwischen Klinikärzten und Niedergelassenen sowie den niedergelassenen Kollegen untereinander auszuloten, um die Kontinuität der evidenz-basierten Therapie zu verbessern.

Bezüglich der nichtmedikamentösen Therapie wäre die Ausprägung regionaler Unterschiede im Impfverhalten bei Herzinsuffizienz sowie Parallelen bezüglich allgemeiner Impfmoral/-müdigkeit eine relevante Forschungsfrage. Weiterhin sollten Gründe für die Probleme, die Patienten mit dem Einhalten der allgemeinen Verhaltensregeln (kochsalzarme Ernährung, tägliches Wiegen) haben, noch genauer untersucht werden. Die Erarbeitung geeigneter Hilfsinstrumente oder Maßnahmen stellt den nächsten Schritt dar. 
Für die Analyse der therapeutischen Langzeitkontinuität nach Entlassung ist wichtig zu wissen, wie lange der günstige Effekt der leitliniengerechteren Therapie im Zuge des Krankenhausaufenthalts nach Entlassung aufrechterhalten bleibt. Unsere Daten zeigten für die Nachbeobachtung 14 Tage nach Entlassung keine signifikante Veränderung in den Verordnungen.

\subsection{Ausblick}

Im Rahmen aktueller gesundheitspolitischer Entwicklungen erhält der zielgerichtete Einsatz von Diagnostik und Therapie einen zunehmenden Stellenwert. Die Nutzung bewährter diagnostischer Mittel und der Einsatz von Medikamenten, die sich in Studien als wirksam, bzw. prognoseverbessernd erwiesen haben, dient einer optimierten Therapie (die auch unter wachsendem ökonomischem Druck zunehmend gefordert wird). Nur so kann eine Patientenbetreuung mit dem Ziel der Sekundär- und Tertiärprävention aufrechterhalten werden. Die kontinuierliche Weiterentwicklung und Aktualisierung von Leitlinien, deren optimierte Implementation sowie die weitere Vernetzung des ambulanten und stationären Versorgungsbereichs wirken in diesem Sinne. Sie können dazu beitragen, die vorhandenen Mittel gezielt und evidenz-basiert einzusetzen, um so am direktesten im Sinne einer verbesserten Patientenbetreuung wirken zu können. 


\section{Zusammenfassung}

\section{Einleitung:}

Trotz nationaler und internationaler Empfehlungen zur medikamentösen und nichtmedikamentösen Behandlung von Patienten mit Herzinsuffizienz erscheint deren Umsetzung im hausärztlichen Versorgungsbereich problematisch. Diese Arbeit untersucht die Umsetzung evidenzbasierter Therapiekriterien im prästationären, stationären und poststationären Vergleich. Im Vordergrund stand dabei die Verordnung von ACEHemmern bzw. AT-II-Antagonisten und Betablockern sowie der Einsatz von Aldosteronantagonisten bei schwerer erkrankten Patienten. Zusätzlich befragten wir die Patienten zu ihrem Verhalten bezüglich nichtmedikamentöser Therapien.

\section{Methoden:}

Bei 91 Patienten mit Herzinsuffizienz wurden während des Aufenthaltes im Universitätsklinikum Göttingen die Medikation des einweisenden Hausarztes, der Krankenhausärzte sowie klinische Charakteristika erhoben. Die poststationäre hausärztliche Medikation erfragten wir durch ein Telefoninterview 14 Tage nach Entlassung. Ferner baten wir die Hausärzte um die Angabe der prä- und poststationären Medikation auf einer Faxvorlage.

\section{Ergebnisse:}

Im Krankenhaus erhielten die untersuchten Patienten signifikant häufiger ACE-Hemmer $(72,5 \%$ vs. $54,9 \% ; p=0,001)$ und geeignete Betablocker - Metoprolol, Bisoprolol, Carvedilol - ( $82,4 \%$ vs. $56,0 \%)$ als vor der stationären Aufnahme. Aldosteronantagonisten wurden während der stationären Behandlung zwar häufiger $(38,5 \%$ vs. $16,5 \%)$ als prästationär verordnet, jedoch nicht in erster Linie bei den schwerer erkrankten Patienten, wie es evidenzbasierte Empfehlungen nahe legen. Der Schweregrad der Herzerkrankung (NYHA-Stadium) beeinflusste die Verordnung von Aldosteronantagonisten weder im hausärztlichen noch im stationären Bereich. Nach Krankenhausentlassung erhielten noch $71,4 \%$ der Patienten die in der Klinik verordneten ACE-Hemmer (77,9\% evidenzbasierte Betablocker und 33,8\% Aldosteronantagonisten). Die Verordnungskonstanz bei den kardialen Medikamenten lag an der Schnittstelle vom Krankenhaus zum Hausarzt mit $67,9 \%$ deutlich über der an der Schnittstelle vom Hausarzt zum Kranken- 
haus, wo nur nahezu ein Drittel der Medikamente beibehalten wurden. Bezüglich des nichtmedikamentösen Therapiemanagements gaben $39,1 \%$ der Patienten an, sich kochsalzarm zu ernähren. 28,7\% beschränkten ihre tägliche Trinkmenge und 42,9\% der Patienten kontrollierten ihr Gewicht mehrmals wöchentlich bis täglich.

\section{Schlussfolgerungen:}

In der Mehrzahl der Fälle erfolgte im Krankenhaus die Gabe von Arzneimitteln nach Kriterien der evidenzbasierten Medizin. Deren Weiterverordnung 14 Tage nach Entlassung blieb weitgehend konstant und ergab somit keine signifikante Diskontinuität in der Verordnung. Es besteht jedoch weiterhin Verbesserungspotenzial in der Umsetzung einer evidenzbasierten Therapie der Herzinsuffizienz. Die weitere Abklärung von Gründen, die einer leitliniengerechten Therapie im Alltag entgegenstehen, ist notwendig. 


\section{Anhang}

\subsection{Dokumentationsbogen}

1. Patientencode:

Station: $\mathrm{Hz}$ :

2. Geschlecht $\square$ männlich $\square$ weiblich

3. Geburtsjahr

4. Name/Adresse des Hausarztes:

\section{FRAGEN AN DEN PATIENTEN:}

NYHA IV Leiden Sie in Ruhe unter Luftnot?

NYHA III Haben Sie Luftnot bei leichter Belastung: Gang zur Toilette/Gehen in der Ebene?

NYHA II Haben Sie Luftnot bei stärkerer Belastung: Treppensteigen/Bergauflaufen?

NYHA I keine Luftnot

5. NYHA-Klassifikation $\quad \square$ I $\quad \square$ II $\square$ III $\square$ IV

6. Wissen Sie an welcher Erkrankung/welchen Erkrankungen Sie leiden?

ja $\square \quad$ nein

Wenn ja, welche?

7. Welcher Arzt betreut Sie ambulant mit Ihrer Herzerkrankung vorwiegend?

(Name, Anschrift, Fachrichtung - fehlendes ggf. aus Ärzteverzeichnis o. Tel.-Buch ergänzen)

8. Wie regelmäßig sind Sie bei diesem Arzt in Betreuung?

\section{regelmäßige, feste Termine unregelmäßige Termine nur bei Bedarf \\ (Termine nur auf Initiative des Pat.)}

9. Ernähren Sie sich kochsalzarm?

10. Kontrollieren Sie Ihre Trinkmenge?

Wenn ja, wie viel trinken Sie etwa täglich?

nie/seltener als 1 mal pro Monat

ca. 1 mal pro Monat

ca. 1 mal pro Woche

mehrmals pro Woche/täglich

11. Wie häufig wiegen Sie sich? nein

nein

ja

Liter

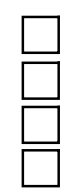

12. Wenn Sie innerhalb von zwei Tagen 2-3 Kilo zunehmen würden, was würden Sie tun?

(Nennt der Patient:

a) „Arzt kontaktieren/aufsuchen“

b) „Wassertabletten/Diuretika erhöhen“

ja

nein

nein

13. Lassen Sie sich jährlich gegen Grippe (Influenza) impfen? ja $\square \quad$ nein 
14. Lassen Sie sich regelmäßig gegen Pneumokokken (Lungenentzündung) impfen?

15. Leiden Sie unter einer Unverträglichkeit oder Allergie gegenüber einem bestimmten Medikament?

nein

Wenn ja, welche?

16. Dürfen Sie aus anderen Gründen bestimmte Medikamente nicht nehmen?

Wenn ja, welche?

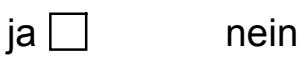

17. Welche Herz-Medikamente haben Sie vor dem Aufenthalt eingenommen?

weiß nicht

Bitte geben Sie auch die Dosis an.

Medikamente/Dosis:

\begin{tabular}{|l|l|l|l|l|}
\hline Medikament & Morgens & Mittags & Abends & zur Nacht \\
\hline & & & & \\
\hline & & & & \\
\hline & & & & \\
\hline & & & & \\
\hline & & & & \\
\hline & & & & \\
\hline & & & & \\
\hline & & & & \\
\hline & & & & \\
\hline
\end{tabular}

Sind diese hier geändert worden?

ja $\square$

nein

weiß nicht

18. Wurde mit Ihnen über die Änderung gesprochen?

ja $\square$

nein

\section{EINWEISUNGSSCHEIN/VERLEGUNGSBRIEF}

19. Einweisung über
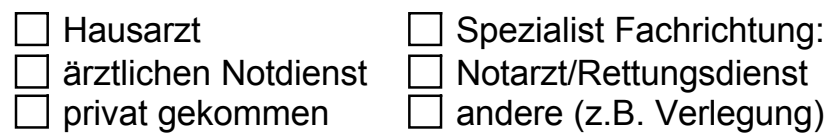

20. Einweisungsdiagnose(n):

(It. Einweisungsschein/Verlegungsbrief)

21. Wurde die Herzinsuffizienz bei Einweisung aufgeführt?

nein

22. Symptome der Herzinsuffizienz:

(bei Einweisung/lt. Aufnahmebefund))

23. Kontraindikationen/Unverträglichkeiten gegen:

(It. Einweisungsschein/Verlegungsbrief) 
24. Medikation bei Einweisung:

-Vormedikation-

(mögl. genauer Präparatename, Dosis und generischer Name)

\begin{tabular}{|l|l|l|l|l|}
\hline Medikament & Morgens & Mittags & Abends & zur Nacht \\
\hline & & & & \\
\hline & & & & \\
\hline & & & & \\
\hline & & & & \\
\hline & & & & \\
\hline & & & & \\
\hline & & & & \\
\hline & & & & \\
\hline
\end{tabular}

\section{AKTE}

25. NYHA-Klassifikation

(It. Aufnahmebefund)

$\square$ I $\square$ II $\square$ III $\square$ IV $\square$ nicht dokumentiert

26. In der Klinik durchgeführte kardiologische Diagnostik:

(It. Akte)

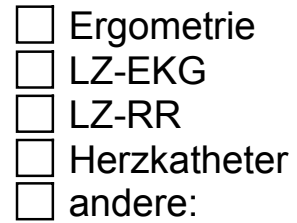

27. Echokardiografie (bei mehreren den letzten Befund)

Wenn ja, Kurzbefund:

28. Ejektionsfraktion: $\%$

29. Aktuelle Medikation bei Erhebung (Med. aus der Planette):

\begin{tabular}{|l|l|l|l|l|}
\hline Medikament & Morgens & Mittags & Abends & zur Nacht \\
\hline & & & & \\
\hline & & & & \\
\hline & & & & \\
\hline & & & & \\
\hline & & & & \\
\hline & & & & \\
\hline & & & & \\
\hline & & & & \\
\hline & & & & \\
\hline
\end{tabular}




\subsection{Fragebogen Telefoninterview}

\section{Telefonische Befragung 14 Tage nach Entlassung}

Patientencode: Datum des Telefonats:

Guten Tag, mein Name ist vom Universitätsklinikum Göttingen. Sie erinnern sich sicher noch, dass Sie während Ihres Klinikaufenthalts an unserer Befragung zur Behandlung der Herzschwäche teilgenommen haben. Wir möchten Ihnen nun einige kurze Fragen stellen, wie Ihre Behandlung nach der Entlassung weitergeführt wurde.

\section{Zunächst einige Fragen zu Ihrer derzeitigen körperlichen Belastbarkeit (NYHA- Klassifikation):}

NYHA IV Leiden Sie in Ruhe unter Luftnot?

NYHA III Haben Sie Luftnot bei leichter Belastung: Gang zur Toilette/Gehen in der Ebene?

NYHA II Haben Sie Luftnot bei stärkerer Belastung: Treppensteigen/Bergauflaufen?

NYHA I keine Luftnot

\section{Ergebnis:}

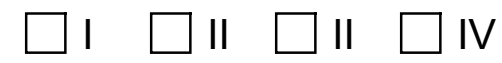

2. Wann sind Sie entlassen worden? Datum:

3. Waren Sie zwischenzeitlich wieder im Krankenhaus? Wenn ja, Grund:

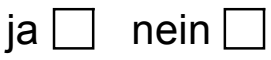

4. Waren Sie nach der Entlassung schon beim Arzt? ja, beim Hausarzt ja, beim Kardiologen ja, andere nein

Wenn ja, wann? Datum:

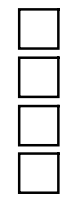

5. Wurden bei diesem Termin weitere Kontrolltermine vereinbart?

$$
\begin{aligned}
& \text { ja, regelmäßige, feste Termine } \\
& \text { (z.B. alle } 2 \text { oder } 4 \text { Wochen) } \\
& \text { ja, der nächste Termin } \\
& \text { nur bei Bedarf } \\
& \text { (Termine nur auf Initiative des Pat.) } \\
& \text { nein }
\end{aligned}
$$

6. Wurde durch den weiterbetreuenden Arzt (z.B. Hausarzt) nach der Entlassung aus der Klinik etwas an Ihrer Medikation geändert?

$$
\text { ja } \square \quad \text { nein } \square \quad \text { weiß nicht }
$$

Wenn ja, wurde mit Ihnen über die Änderung gesprochen?

ja nein

Warum wurde etwas geändert? 
7. Welche Medikamente nehmen Sie zurzeit ein?

weiß nicht

Bitte geben Sie auch die Dosis an.

Medikamente/Dosis:

\begin{tabular}{|l|l|l|l|l|}
\hline Medikament & Morgens & Mittags & Abends & zur Nacht \\
\hline & & & & \\
\hline & & & & \\
\hline & & & & \\
\hline & & & & \\
\hline & & & & \\
\hline & & & & \\
\hline & & & & \\
\hline & & & & \\
\hline & & & & \\
\hline
\end{tabular}

8. Ich nenne nun einige allgemeine Maßnahmen, die bei der Behandlung von Herzerkrankungen unter Umständen eine Rolle spielen können. Bitte geben Sie an, ob die jeweilige Maßnahme beim Klinikaufenthalt bzw. danach mit Ihnen besprochen wurde und wenn ja, von wem (Klinikarzt oder weiterbetreuender Arzt z.B. Hausarzt).

$\begin{array}{ll}\text { nicht } & \text { vom Klinikarzt } \\ \text { besprochen } & \text { besprochen }\end{array}$

Kochsalzarme Ernährung

Kontrolle der täglichen Trinkmenge

Kontrolle des Körpergewichts durch häufiges Wiegen

Aufsuchen oder Informieren des

Arztes bei

starker Gewichtszunahme

(z.B. mehr als $2 \mathrm{~kg}$ in 2 Tagen) vom Hausarzt/ weiterbehandelnden

Arzt besprochen

Wir haben jetzt alle Fragen bearbeitet, haben Sie selbst noch Fragen oder Anmerkungen?

Vielen herzlichen Dank für Ihre Mitarbeit, wir melden uns in etwa 6 Monaten wieder bei Ihnen! 


\subsection{Fax an Hausärzte}

Fragebogen für den Hausarzt

„Pharmakotherapie der Herzinsuffizienz

an der stationär-ambulanten Schnittstelle

(poststationäre Verlaufsbeobachtung),,

Patientendaten:

Name:

Vorname:

Geburtsdatum:

Stationärer Aufenthalt:

Bitte geben Sie hier die Medikation an, die der Patient vor seinem stationären Aufenthalt von Ihnen erhalten hat. Soweit dies möglich ist bitte auch mit genauer Dosierung.

\begin{tabular}{|l|l|l|l|l|}
\hline Medikament & Morgens & Mittags & Abends & zur Nacht \\
\hline & & & & \\
\hline & & & & \\
\hline & & & & \\
\hline & & & & \\
\hline & & & & \\
\hline & & & & \\
\hline & & & & \\
\hline & & & & \\
\hline & & & & \\
\hline
\end{tabular}

Bitte geben Sie hier die Medikation an, die der Patient nach seinem stationären Aufenthalt von Ihnen erhalten hat. Soweit dies möglich ist, bitte auch hier die genaue Dosierung angeben.

\begin{tabular}{|c|c|c|c|c|}
\hline Medikament & Morgens & Mittags & Abends & zur Nacht \\
\hline & & & & \\
\hline & & & & \\
\hline & & & & \\
\hline & & & & \\
\hline & & & & \\
\hline & & & & \\
\hline & & & & \\
\hline & & & & \\
\hline & & & & \\
\hline 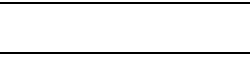 & & & & \\
\hline
\end{tabular}




\section{Literaturverzeichnis}

ACC/AHA Task Force on Practice Guidelines (2001): ACC/AHA guidelines for the evaluation and management of chronic heart failure in the adult: Executive summary. Circulation 104, 2996-3007

Adlam D, Silcocks P, Sparrow N (2005): Using BNP to develop a risk score for heart failure in primary care. Eur Heart J $\underline{26}, 1086-1093$

Ajani UA, Ford ES, Mokdad AH (2005): Examining the coverage of influenza vaccination among people with cardiovascular disease in the United States. Am Heart J $\underline{149}$, 254-259

al Mahdy HA, Seymour DG (1990): How much can elderly patients tell us about their medications? Postgrad Med J $\underline{66}, 116-121$

Arzneimittelkommission der deutschen Ärzteschaft: Chronische Herzinsuffizienz. Empfehlung zur Therapie der chronischen Herzinsuffizienz. Gemeinsame Empfehlung mit der Gesellschaft für Kardiologie - Herz - und Kreislaufforschung. AVP-Sonderheft Therapieempfehlungen, 2. Auflage, Arzneimittelkommission der deutschen Ärzteschaft, Köln 2001

Baker DW, Hayes RP, Massie BM, Craig CA (1999): Variations in family physicians' and cardiologists care for patients with heart failure. Am Heart J $\underline{138}, 826-34$

Beers MH, Dang J, Hasegawa J, Tamai IY (1989): Influence of hospitalization of drug therapy in the elderly. J Am Geriatr Soc 37, 679-683

Bentley B, De Jong MJ, Moser DK, Peden AR (2005): Factors related to nonadherence to low sodium diet recommendations in heart failure patients. Eur $\mathrm{J}$ Cardiovasc Nurs $\underline{4}$, 331-336 
Bijl D, Van Sonderen E, Haaijer-Ruskamp FM (1998): Prescription changes and drug costs at the Interface between primary and specialist care. Eur J Clin Pharmacol $\underline{54}$, 333-336

Böger RH (2002): Wie wird die chronische Herzinsuffizienz heute tatsächlich behandelt? Dtsch Med Wochenschr 127, 1764-1768

Bungard TJ, McAlister FA, Johnson JA, Tsuyuki RT (2001): Underutilisation of ACE inhibitors in patients with congestive heart failure. Drugs $\underline{61}, 2021-2033$

Burnier M, Brunner HR (2000): Angiotensin II receptor antagonists. Lancet $\underline{355}$, 637645

Butler J, Weingarten JP Jr, Weddle JA, Jain MK (2003): Differences among hospitals in delivery of care for heart failure. J Health Qual $\underline{25}$, 4-10

Chin MH, Friedmann PD, Cassel CK, Lang RM (1997): Differences in generalist and specialist physicians' knowledge and use of angiotensin-converting enzyme inhibitors for congestive heart failure. J Gen Intern Med 12, 523-530

CIBIS-II-Research-Group (1999): The Cardiac Insufficiency Bisoprolol Study II (CIBISII): a randomised trial. Lancet $\underline{353}, 9-13$

Cleland JG, Cohen-Solal A, Aguilar JC, Dietz R, Follath F, Freemantle N, Gavazzi A, van Gilst WH, Hobbs FD, Korewicki J, et al. (2002): Management of heart failure in primary care (the IMPROVEMENT of heart failure Programme): an international survey. Lancet $\underline{360}, 1631-1639$

Cline CM, Boman K, Holst M, Erhardt LR; Swedish Society of Cardiology Working Group for Heart Failure (2002): The management of heart failure in Sweden. Eur J Heart Fail $\underline{4}$, 373-6

Cochrane RA, Mandal AR, Ledger-Scott M, Walker R (1992): Changes in drug treatment after discharge from hospital in geriatric patients. BMJ $\underline{305}, 694-696$ 
Cohn JN, Johnson G, Ziesche S, Cobb F, Francis G, Tristani F, Smith R, Dunkman WB, Loeb H, Wong M, et al. (1991): A comparison of enalapril with hydralazine-isosorbide dinitrate in the treatment of chronic congestive heart failure. N Engl J Med $\underline{325}, 303-310$

Cohn JN, Tognoni G, Glazer RD, Spormann D, Hester A (1999): Rationale and design of the Valsartan Heart Failure Trial: a large multinational trial to assess the effects of valsartan, an angiotensin-receptor blocker, on morbidity and mortality in chronic congestive heart failure. J Card Fail $\underline{5}, 155-160$

Cox JL, Ramer SA, Lee DS, Humphries K, Pilote L, Svenson L, Tu JV, Canadian cardiovascular outcomes research team investigators (2005): Pharmacological treatment of congestive heart failure in Canada: a description of care in five provinces. Can J Cardiol $\underline{21}, 337-343$

de Vries CS, van Diepen NM, Tromp TF, de Jong-van den Berg LT (1996): Auditing GP's prescribing habits: cardiovascular prescribing frequently continues medication initiated by specialists. Eur J Clin Pharmacol $\underline{50}$, 349-352

DIMDI: ICD-10 Diagnosenthesaurus Version 3.0, Stand Januar 2000, Deutscher ÄrzteVerlag, Köln 1999

Doughty RN, Wright SP, Pearl A, Walsh HJ, Muncaster S, Whalley GA, Gamble G, Sharpe N (2002): Randomized, controlled trial of integrated heart failure management: The Auckland Heart Failure Management Study. Eur Heart J 로, 139-146

Epstein MD (1995): Communication between primary care physicians and consultants. Arch Fam Med $\underline{4}, 403-409$

Erdmann E, Lechat P, Verkenne P, Wiemann H (2001): Results from post-hoc analyses of the CIBIS II trial: effect of bisoprolol in high-risk patient groups with chronic heart failure. Eur J Heart Fail $\underline{3}$, 469-479

Flather MD, Yusuf S, Kober L, Pfeffer M, Hall A, Murray G, Torp-Pedersen C, Ball S, Pogue J, Moye L, et al. (2000): Longterm ACE-inhibitor therapy in patients with heart 
failure or left-ventricular dysfunction: a systematic overview of data from individual patients. ACE-Inhibitor Myocardial Infarction Collaborative Group. Lancet $\underline{355}, 1575-1581$

Fuat A, Hungin APS, Murphy JJ (2003): Barriers to accurate diagnosis and effective management of heart failure in primary care: qualitative study. BMJ $\underline{326}, 196$

Gnani S, Gray J, Khunti K, Majeed A (2004): Managing heart failure in primary care: first steps in implementing the National Service Framework. J Public Health $\underline{26}, 42-47$

Greer AL (1988): The state of the art versus the state of the science. The diffusion of new medical technologies into practice. Int J Technol Assess Health Care 4, 5-26

Grigorian Shamagian L, Varela Roman A, Virgos Lamela A, Rigueiro Veloso P, Garcia Acuna JM, Gonzalez-Juanatey JR (2005): Long-term trends in drug prescription for hospitalized patients with congestive heart failure. Influence of type of dysfunction. Rev Esp Cardiol $\underline{58}$, 381-388

Hakansson A, Andersson H, Cars H, Melander A (2001): Prescribing, prescription costs and adherence to formulary committee recommendations: long-term differences between physicians in public and private care. Eur J Clin Pharmacol $\underline{57}$, 65-70

Hall AS, Murray GD, Ball SG (1997): Follow-up study of patients randomly allocated ramipril or placebo for heart failure after acute myocardial infarction: AIRE Extension (AIREX) Study. Acute Infarction Ramipril Efficacy. Lancet 349, 1493-1497

Harder S, Fischer P, Krause-Schafer M, Ostermann K, Helms G, Prinz H, Hahmann M, Baas H (2005): Structure and markers of appropriateness, quality and performance of drug treatment over a 1-year period after hospital discharge in a cohort of elderly patients with cardiovascular diseases from Germany. Eur J Clin Pharmacol $\underline{60}$, 797-805

Hickling JA, Nazareth I, Rogers S (2001): The barriers to effective management of heart failure in general practice. Br J Gen Pract $\underline{51}$, 615-618 
Himmel W, Tabache M, Kochen MM (1996): What happens to long-term medication when general practice patients are referred to hospital? Eur J Clin Pharmacol $\underline{50}$, 253257

Himmel W, Kochen MM, Sorns U, Hummers-Pradier E (2004): Drug changes at the interface between primary and secondary care. Int J Clin Pharmacol Ther $\underline{42}, 103-109$

Hjalmarson A, Goldstein S, Fagerberg B, Wedel H, Waagstein F, Kjekhus J, Wikstrand J, El Allaf D, Vitovec J, Aldershvile J, et al. (2000): Effects of controlled-release metoprolol on total mortality, hospitalizations, and well-being in patients with heart failure: the Metoprolol CR/XL Randomized Intervention Trial in congestive heart failure (MERIT-HF). MERIT-HF Study Group. JAMA $\underline{283}, 1295-1302$

Hood WB Jr, Youngblood M, Ghali JK, Reid M, Rogers WJ, Howe D, Teo KK, LeJentel TH (1991): Initial blood pressure response to enalapril in hospitalized patients (Studies of Left Ventricular Dysfunction [SOLVD]). Am J Cardiol $\underline{68}, 1465-1468$

Hoppe UC (2005): Wie erkenne ich eine beginnende Herzinsuffizienz? MedRev $\underline{6}$, 8-10

Hoppe UC, Erdmann E, für die Kommission für Klinische Kardiologie, Deutsche Gesellschaft für Kardiologie - Herz - und Kreislaufforschung (2001): Leitlinien zur Therapie der chronischen Herzinsuffizienz. Z Kardiol $\underline{90}$, 218-241

Horne R, Coombes I, Davies G, Hankins M, Vincent R (1999): Barriers to optimum management of heart failure by general practitioners. Br J Gen Pract $\underline{49}$, 353-357

ISIS-4 Collaborative Group (1995): ISIS-4: randomised factorial trial assessing early oral captopril, oral mononitrate, and intravenous magnesium sulphate in 58,050 patients with suspected acute myocardial infarction. Lancet $\underline{345}, 669-685$

Kanouse DE, Jacoby I (1988): When does information change practitioners' behavior? Int J Technol Assess Health Care 4, 27-33 
Kasje WN, Dening P, Stewart RE, de Graeff PA, Haaijer-Ruskamp FM (2005): Physician, organisational and patient characteristics explaining the use of angiotensin converting enzyme inhibitors in heart failure treatment: a multilevel study. Eur J Clin Pharmacol $\underline{61}, 145-151$

Kober L, Torp-Pedersen C, Carlsen JE, Bagger H, Eliasen P, Lyngborg K, Videbaek J, Cole DS, Auclert L, Pauly NC (1995): For the Trandolapril Cardiac Evaluation (TRACE) Study Group: A clinical trial of the angiotensin-converting-enzyme inhibitor trandolapril in patients with left ventricular dysfunction after myocardial infarction. N Engl J Med $\underline{333}$, 1670-1676

Komajda M, Follath F, Swedberg K, Cleland J, Aguilar JC, Cohen-Solal A, Dietz R, Gavazzi A, Van Gilst WH, Hobbs R, et al.; Study Group on Diagnosis of the Working Group on Heart Failure of the European Society of Cardiology (2003): The EuroHeart Failure Survey programme - a survey on the quality of care among patients with heart failure in Europe. Part 2: treatment. Eur Heart J 24, 464-474

Komajda M, Lapuerta P, Hermans N, Gonzalez-Juanatey JR, van Veldhuisen DJ, Erdmann E, Tavazzi L, Poole-Wilson P, Le Pen C (2005): Adherence to guidelines is a predictor of outcome in chronic heart failure: the MAHLER survey. Eur Heart $\mathrm{J} \underline{26}, 1653-$ 1659

Lechat P, Packer M, Chalon S, Cucherat M, Arab T, Boissel JP (1998): Clinical effects of beta-adrenergic blockade in chronic heart failure: a meta-analysis of double-blind, placebo-controlled, randomized trials. Circulation $\underline{98}, 1184-1191$

Lee DS, Austin PC, Rouleau JL, Liu PP, Naimark D, Tu JV (2003): Predicting mortality among patients hospitalized for heart failure. Derivation and validation of a clinical model. JAMA 290, 2581-2587

Lowe J, Candlish P, Henry D, Wlodarcyk J, Fletcher P (2000): Specialist or generalist care? A study of the impact of a selective admitting policy for patients with cardiac failure. Int J Qual Health Care $\underline{12}$, 339-345 
Maison P, Cunin P, Hemery F, Fric F, Elie N, Del'volgo A, Dubois-Rande JL, Hittinger L, Macquin-Mavier I (2005): Utilisation of medications recommended for chronic heart failure and the relationship with annual hospitalisation duration in patients over 75 years of age. A pharmacoepidemiological study. Eur J Clin Pharmacol $\underline{61}$, 445-451

Martensson J, Stromberg A, Dahlstrom U, Karlsson JE, Fridlund B (2005): Patients with heart failure in primary health care: effects of a nurse-led intervention on health-related quality of life and depression. Eur J Heart Fail $\underline{7}$, 393-403

Masoudi FA, Krumholz HM (2003): Polypharmacy and comorbidity in heart failure. BMJ $\underline{327}, 513-514$

Masoudi FA, Rathore SS, Wang Y, Havranek EP, Curtis JP, Foody JM, Krumholz HM (2004): National patterns of use and effectiveness of angiotensin-converting enzyme inhibitors in older patients with heart failure and left ventricular systolic dysfunction. Circulation $\underline{110}, 724-731$

McColl A, Smith H, White P, Field J (1998): General practitioners' perceptions of the route to evidence based medicine: a questionnaire survey. BMJ $\underline{316}, 361-365$

McKelvie RS, Yusuf S, Pericak D, Avezum A, Burns RJ, Probstfield J, Tsuyuki RT, White M, Rouleau J, Latini R, et al. (1999): Comparison of candesartan, enalapril, and their combination in congestive heart failure: randomized evaluation of strategies for left ventricular dysfunction (RESOLVD) pilot study. The RESOLVD Pilot Study Investigators. Circulation $\underline{100}, 1056-1064$

McMullan R, Silke B (2001): A survey of the dose of ACE inhibitors prescribed by general physicians for patients with heart failure. Postgrad Med J $\underline{77}, 765-768$

McMurray J, Stewart S (2000): Epidemiology, aetiology, and prognosis of heart failure. Heart $\underline{83}, 596-602$

McMurray J, Cohen-Solal A, Dietz R, Eichhorn E, Erhardt L, Hobbs R, Maggioni A, Pina I, Soler-Soler J, Swedberg K ; Clinical Research Initiative in Heart failure (2001): Practi- 
cal recommendations for the use of ACE inhibitors, beta-blockers and spironolactone in heart failure: putting guidelines into practice. Eur J Heart Fail $\underline{3}$, 495-502

MERIT-HF Study Group (1999): Effect of metoprolol CR/XL in chronic heart failure: Metoprolol CR/XL Randomised Intervention Trial in Congestive Heart Failure. (MeritHF). Lancet $\underline{353}, 2001-2007$

Mottur- Pilson C, Snow V, Bartlett K (2001): Physician explanation for failing to comply with "best practices". Eff Clin Pract $\underline{4}$, 207-213

Muntwyler J, Follath F (2000): Medical treatment of heart failure: an analysis of actual treatment practices in outpatients in Switzerland. The Swiss "IMPROVEMENT of HF" Group. Schweiz Med Wochenschr 130, 1192-1198

Ni H, Naumann D, Burgess D, Wise K, Crispell K, Hershberger RE (1999): Factors influencing knowledge of and adherence to self-care among patients with heart failure. Arch Intern Med 159, 1613-1619

Nilsson G, Strender LE (2002): Management of heart failure in primary health care, a retrospective study on electronic patient records in a registered population. Scand $\mathrm{J}$ Prim Health Care 20, 161-165

Nohria A, Chen Y, Morton DJ, Walsh R, Vlasses PH, Krumholz HM (1999): Quality of care for patients hospitalized with heart failure at academic medical centers. Am Heart $J$ $\underline{137}, 1028-1034$

O'Meara F, Solomon S, McMurray J, Pfeffer M, Yusuf S, Michelson E, Granger C, Olofsson B, Young JB, Swedberg K (2004): Effect of candesartan on New York Heart Association functional class. Results of the Candesartan in Heart failure: Assessment of Reduction in Mortality and morbidity (CHARM) programme. Eur Heart J $\underline{25}$, 1920-1926

Omori DM, Potyk RP, Kroenke K (1991): The adverse effects of hospitalization on drug regimens. Arch Intern Med 151, 1562-1564 
Packer M, Poole-Wilson PA, Armstrong PW, Cleland JG, Horowitz JD, Massie BM, Ryden L, Thygesen K, Uretsky BF (1999): Comparative effects of low and high doses of the angiotensin-converting enzyme inhibitor, lisinopril, on morbidity and mortality in chronic heart failure. ATLAS Study Group. Circulation 100, 2312-2318

Packer M, Fowler MB, Roecker EB, Coats AJ, Katus HA, Krum H, Mohacsi P, Rouleau JL, Tendera M, Staiger C, et al.; Carvedilol Prospective Randomized Cumulative Survival (COPERNICUS) Study Group (2002): Effect of carvedilol on the morbidity of patients with severe chronic heart failure: results of the carvedilol prospective randomized cumulative survival (COPERNICUS) study. Circulation 106, 2194-2199

Pfeffer MA, Braunwald E, Moye LA, Basta L, Brown EJ Jr, Cuddy TE, Davis BR, Geltman EM, Goldman S, Flaker GC, et al. (1992): Effect of captopril on mortality in patients with left ventricular dysfunction after myocardial infarction. Results of the survival and ventricular enlargement trial. The SAVE Investigators. N Engl J Med $\underline{327}, 669-677$

Phillips SM, Marton RL, Tofler GH (2004): Barriers to diagnosing and managing heart failure in primary care. Med $\mathrm{J}$ Aust $\underline{181}, 78-81$

Pitt B, Zannad F, Remme WJ, Cody R, Castaigne A, Perez A, Palensky J, Wittes J (1999): The effect of spironolactone on morbidity and mortality in patients with severe heart failure. Randomized Aldactone Evaluation Study Investigators. N Engl J Med $\underline{34}$, 709-717

Pitt B, Poole-Wilson PA, Segal R, Martinez FA, Dickstein K, Camm AJ, Konstam MA, Riegger G, Klinger GH, Neaton J, et al. (2000): Effect of losartan compared with captopril on mortality in patients with symptomatic heart failure: randomised trial - the Losartan Heart Failure Survival Study ELITE II. Lancet $\underline{355}$, 1582-1587

Polanczyk CA, Newton C, Dec GW, Di Salvo TG (2001): Quality of care and hospital readmission in congestive heart failure: an explicit review process. J Card Fail $\underline{7}$, 289298 
Ramires FJ, Mansur A, Coelho O, Maranhao M, Gruppi CJ, Mady C, Ramires JA (2000): Effect of spironolactone on ventricular arrhythmias in congestive heart failure secondary to idiopathic dilated or to ischemic cardiomyopathy. Am J Cardiol $\underline{85}, 1207-$ 1211

Rutten FH, Grobbee DE, Hoes AW (2003): Differences between general practitioners and cardiologists in diagnosis and management of heart failure: a survey in every-day practice. Eur J Heart Fail $\underline{5}$, 337-344

Schmedtje JF, Evans GW, Byerly W, King M, Goonan K, Blastock-Glenn C, Croft JB, Goff DC Jr (2003): Treatment of chronic heart failure in a managed care setting. Baseline results from the Achieving Cardiac Excellence Project. N C Med J $\underline{64}, 4-10$

Task Force for the Diagnosis and Treatment of Chronic Heart Failure, European Society of Cardiology (2001): Guidelines for the diagnosis and treatment of chronic heart failure. Eur Heart J 22, 1527-1560

The CONSENSUS Trial Study Group (1987): Effects of enalapril on mortality in severe congestive heart failure. Results of the Cooperative North Scandinavian Enalapril Survival Study (CONSENSUS). N Engl J Med $\underline{316}, 1429-1435$

The SOLVD Investigators (1991): Effect of enalapril on survival in patients with reduced left ventricular ejection fractions and congestive heart failure. N Engl J Med $\underline{325}$, 293302

Tomson Y, Wessling A, Tomson G (1994): General practitioners for rational use of drugs. Examples from Sweden. Eur J Clin Pharmacol 4ㄱ, 213-219

Wagdi P, Vuilliomenet A, Kaufmann U, Richter M, Bertel O (1993): Ungenügende Behandlungsdisziplin, Patienteninformation und Medikamentenverschreibung als Ursachen für die Notfallhospitalisation bei chronisch herzinsuffizienten Patienten. Schweiz Med Wochenschr 123, 108-112 
Weil E, Tu JV (2001): Quality of congestive heart failure treatment at a Canadian teaching hospital. CMAJ $\underline{165}, 284-287$

Weltermann B, Martin C, Adl S, Küching A, Korbonits G, Höpp HW (1997): Verordnungspraxis von Betablockern an der Schnittstelle von stationärer zu ambulanter Versorgung. Gesundheitswesen $\underline{59}, 258-261$

Wikstrand J, Wedel H, Ghali J, Deedwania P, Fagerberg B, Goldstein S, Gottlieb S, Hjalmarson A, Kjekshus J, Waagstein F (2003): How should subgroup analyses affect clinical practice? Insight from the Metoprolol succinate controlled-releaesed/extendedrelease randomized intervention trial in heart failure (MERIT-HF). Cardiac Elektrophysiol $\operatorname{Rev} \underline{7}, 264-275$

www.dgk.org. Leitlinien zur Therapie der chronischen Herzinsuffizienz. Stand 07.07.2005

www.evidence.de. Universität Witten/Herdecke. Leitlinie Diagnose und Therapie der chronischen Herzinsuffizienz. Stand 15.01.2005

www.klinikum.uni-heidelberg.de HeiCare - Sektorübergreifende Harmonisierung der Arzneimitteltherapie. Stand 03.01.2006

www.nice.org.uk. Chronic heart failure: Management of chronic heart failure in adults in primary and secondary care. Stand 11.07.2005

www.pmvforschungsgruppe.de, Hausärztliche Leitlinie chronische Herzinsuffizienz Version 2.04. Stand 14.08.2006

www.uni-duesseldorf.de/WWW/AWMF. Therapie der chronischen Herzinsuffizienz. Stand 23.02.2005 


\section{Danksagung}

Danken möchte ich allen, die mich vielfältig unterstützt haben, dass diese Arbeit gelang. Insbesondere danke ich Herrn Prof. Dr. med. Michael M. Kochen für die Überlassung des Themas und die freundliche Unterstützung. Herrn Dr. med. Martin Scherer und Dr. med. Dirk Wetzel verdanke ich eine kritische und zielstrebige Betreuung. Vielen Dank für die wertvolle Unterstützung und die Anleitung zum wissenschaftlichen Arbeiten. Frau Claudia Tomala danke ich für die unermüdliche Hilfe bei der Datenerhebung und Datenerfassung.

Vielen Dank den Mitarbeitern der Abteilung Allgemeinmedizin und der Abteilung Kardiologie, die mich in der Zeit meiner Promotion begleitet haben. 


\section{Curriculum vitae}

Mein Name ist Cordula Sobek. Ich wohne in der Von-Bernuth-Str. 92, 59821 Arnsberg. Am 19.06.1979 wurde ich als Tochter von Dr. med. Hans-Wilhelm Sobek (praktischer Arzt) und Gesine Sobek, geb. Sims (Diplompädagogin), in Halle/Saale geboren. Meine Schulzeit beendete ich 1998 mit dem Abitur an der Latina August-Hermann-Francke in Halle.

Im Rahmen eines freiwilligen sozialen Jahres arbeitete ich bis 1999 in der häuslichen Krankenpflege des Deutschen Roten Kreuzes und nahm im Sommer 1999 das Studium der Humanmedizin an der Georg-August-Universität Göttingen auf. Die ärztliche Vorprüfung legte ich im März 2001 und den ersten Abschnitt der ärztlichen Prüfung im April 2002 ab. Im September 2004 absolvierte ich den zweiten Abschnitt der ärztlichen Prüfung und beendete im November 2005 mein Studium mit dem dritten Abschnitt der ärztlichen Prüfung und dem Erhalt der Approbation als Ärztin.

In der Abteilung Allgemeinmedizin der Georg-August-Universität Göttingen begann ich 2002 meine Promotion mit dem Thema „Evidenz-basierte Pharmakotherapie der Herzinsuffizienz an der stationär-ambulanten Schnittstelle“. Begleitend war ich von 2003 2004 in der Abteilung Allgemeinmedizin als studentische Hilfskraft im Bereich Lehre und Forschungsarbeiten tätig.

Seit dem 01.04.2006 bin ich als Assistenzärztin in der Abteilung Innere Medizin des Karolinen-Hospitals Hüsten tätig. 